Pacific Journal of Mathematics

SMITH EQUIVALENCE FOR FINITE ABELIAN GROUPS

KarL Heinz Dover mann and Dong Your Sum 


\title{
SMITH EQUIVALENCE FOR FINITE ABELIAN GROUPS
}

\author{
Karl Heinz Dovermann and Dong Youp SuH \\ Dedicated to Professor Y. Y. Oh on his 60th birthday
}

\begin{abstract}
For certain even order cyclic and some non-cyclic abelian groups $G$ we construct smooth actions on homotopy spheres $\Sigma$ with exactly two fixed points, $\Sigma^{G}=\{p, q\}$, such that the tangential representations $T_{p} \Sigma$ and $T_{q} \Sigma$ are not isomorphic.
\end{abstract}

1. Introduction. Two real representations $V$ and $W$ of a finite group $G$ are called Smith equivalent if there is a closed smooth manifold $\Sigma$ which is homotopy equivalent to a sphere and $G$ acts smoothly and effectively on $\Sigma$ with exactly two fixed points, $\Sigma^{G}=\{p, q\}$, such that the tangent spaces $T_{p} \Sigma$ and $T_{q} \Sigma$ at $p$ and $q$ are isomorphic to $V$ and $W$ as representations of $G$. Several authors have studied the question of which groups do and which groups do not have non-isomorphic Smith representations. We shall recall their results below.

In this paper we want to contribute two other classes of groups which have non-isomorphic Smith equivalent representations. The first one is as follows. Let $H$ be a cyclic group of odd order, and $G=H \times \mathbb{Z}_{2^{k}}, k \geq 0$. Below we shall give a list of conditions (see Condition 2.2) for a pair $(A, B)$ of representations of $H$. There are cyclic groups $H$ of odd order and non-isomorphic representations $A$ and $B$ of $H$ such that these conditions are satisfied. In Theorem 2.3 and Theorem 2.4 we quote two results from [DP2] and [DW] which provide examples. Theorem B of [DP2] shows that such groups $H$ have non-isomorphic Smith equivalent representations. In $\S 4$ we will prove our first principal result, which extends the just quoted theorem.

TheOREM A. Suppose $H$ is a cyclic group of odd order which has non-isomorphic representations satisfying the conditions in 2.2. Assume that the order of $H$ is divisible by at least three distinct primes. Then $G=H \times \mathbb{Z}_{2^{k}}$ has non-isomorphic Smith equivalent representations.

In particular, if $A$ and $B$ are two representations of $H$ which satisfy Condition 2.2, then there exists an action of $G$ on a homotopy sphere $\Sigma$ with exactly two fixed points $x$ and $y$, and $T_{x} \Sigma-T_{y} \Sigma=l(A-B)$ 
for some $l \neq 0$. Here $A$ and $B$ are considered as representations of $G$ with trivial action of $\mathbb{Z}_{2^{k}}$. If $A \neq B$ then $T_{x} \Sigma \neq T_{y} \Sigma$.

We give the details of the proof only in the case $k=1$. For $k>1$ more notation is needed but the argument is basically the same.

In our second result we consider abelian groups $G$ with at least three non-cyclic Sylow subgroups. Let $A$ and $B$ be real representation of $G$ such that

Condition. 1.1. (1) $A^{K}=B^{K}=0$ whenever $G / K$ is of prime power order.

(2) $\operatorname{Res}_{P} A \cong \operatorname{Res}_{P} B$ whenever $P \subset G$ is of prime power order.

(3) $\operatorname{dim} A^{K}=\operatorname{dim} B^{K}$ for all $K \subset G$.

We shall show in Lemma 5.1 how to construct non-isomorphic representations $A$ and $B$ for any such group $G$. Our second principal result generalizes one of T. Petrie [P1] which we recall below.

Theorem B. Suppose $G, A$ and $B$ as in Condition 1.1. There exists an action of $G$ on a homotopy sphere $\Sigma$ with exactly two fixed points $x$ and $y$ such that $T_{x} \Sigma-T_{y} \Sigma$ is a non-zero multiple of $A-B$. In particular, if $A \neq B$ then $T_{x} \Sigma \neq T_{x} \Sigma$.

This is not only an improvement of Petrie and Randall's result, but we also give a proof which shows that $\Sigma$ may be chosen to be equivariantly cobordant to a product of surfaces as they are constructed in $\S 3$ of [DP2]. (See also $\S 3$ of this paper.) This will be used in [DKS]. In the proof of Theorem B we will use information about a surgery obstruction group for which we thank A. Bak.

The study of Smith equivalent representations is motivated by a question of P. A. Smith [Sm] who asked whether Smith equivalent representations are linearly isomorphic. Atiyah-Bott $[\mathbf{A B}]$ and Milnor [M] established an affirmative answer to the question for semi-free actions and for actions of cyclic groups of odd prime power order. By definition, a semi-free action has the whole group and the trivial group as its only isotropy groups. Sanchez $[\mathbf{S z}]$ showed that the answer is also affirmative for cyclic groups of order $p q$, where $p$ and $q$ are odd primes. Additional elementary considerations show that the answer is affirmative for any group whose order is a product of two primes. Bredon [B] showed for 2-groups that Smith equivalent representations are isomorphic if their dimension is large in comparison to 
the order of the group. Two Smith equivalent representations are said to be $s$-Smith equivalent if the action is semi-linear, i.e., we require in addition that the fixed point sets $\Sigma^{K}$ are homotopy spheres for every subgroup $K \subset G$. Sanchez's result also implies that s-Smith equivalent representations of any cyclic group of odd order are isomorphic.

Petrie announced the first negative answer to Smith's question [P1], see [PR1] for the details of the proof,

THEOREM. Suppose $G$ is an odd order abelian group with at least four non-cyclic Sylow subgroups. There are non-isomorphic Smith equivalent representations of $G$.

In this reference Petrie also posed the problem of finding all groups which have non-isomorphic Smith equivalent representations. Our Theorem $\mathrm{A}$ is a contribution to the solution of this problem. Since Petrie's announcement several authors provided classes of groups which have non-isomorphic s-Smith equivalent representations. One such class are cyclic groups of order $4 m$, where $m>1$. See the work of Cappell-Shaneson [CS1], Petrie [P2], Siegel [Si], and Dovermann [D]. Non-isomorphic s-Smith equivalent representations were also constructed by Suh [Su1] for some non-cyclic abelian groups and by Cho [C1] and [C2] for certain quaternion and dihedral groups. Non-isomorphic Smith equivalent representations of odd order cyclic groups were constructed by Dovermann-Petrie [DP2]. The groups were of rather large orders. Dovermann-Washington [DW] showed that such non-isomorphic Smith equivalent representations also exist for cyclic groups of small orders. The topic of Smith equivalent representations was surveyed in [DPS], [MP], [CS2], and Petrie-Randall [PR2] wrote a book about it.

This review of the history shows that, basically, Petrie's question has been answered for cyclic groups, except for those groups whose order is of the form $2 m$ where $m$ is odd, and this is the class of groups we are treating in this paper. In Theorem $\mathrm{A}$ this is the case when $k=1$. In case $k>1$ the result is interesting for the discussion in [DKS], because we get some additional conclusion based on the specific construction. There we conclude that the actions described in Theorems A and B can be chosen to be real algebraic.

In the construction of s-Smith equivalent representations for groups $\mathbb{Z}_{4 m}$, with $m>1$, the papers mentioned above use in an essential way that the subgroup $\mathbb{Z}_{2 m}$ occurs as isotropy group. This implies that the $\mathrm{s}-\mathrm{Smith}$ equivalent representations restrict to the same representation 
of $\mathbb{Z}_{2 m}$. This is not the case in [Su1], [C1], and this paper. Here one supposes non-isomorphic Smith equivalent representations of the group $H$, and then one uses them to construct such representations for the group $G$ in which $H$ is an index 2 subgroup. In fact, if $V$ and $W$ are Smith equivalent representations of a cyclic group $G$, and $H$ is an index 2 subgroup which is an isotropy group of either $V$ or $W$, then $V$ and $W$ are isomorphic (see [Su2]). Thus the non-isomorphic representations of $G$ in Theorem A must also be non-isomorphic as $H$ representations.

Based on the different constructive approaches it happened that the technique of proof implied if one constructed non-isomorphic (s-) Smith equivalent representations for the group $H$, then one could also construct such examples for the group $G$ in which $H$ is a subgroup. These groups $G$ had to be again of the same form as those groups one started out with. In [PR1] one would assume that $H$ and $G$ are abelian of odd order with at least four non-cyclic Sylow subgroups. In [DP2] and [DW] one would assume that $H$ and $G$ are cyclic of odd order and that $H$ has non-isomorphic representations which satisfy 2.2. More generally we like to conjecture:

Conjecture. Let $H$ be a subgroup of $G$. If $H$ has non-isomorphic Smith equivalent representations, then so does $G$.

2. Preliminary material. We shall formulate Condition 2.2 which is the essential assumption in our Theorem A, and we shall describe how to satisfy it. Let us fix some notation. For any group $G$ we denote by $\mathscr{P}(G)$ the set of all subgroups of $G$ of prime power order. Also, let $\mathscr{P}$ denote the set of all groups of prime power order. We use the following standard notation for the complex 1-dimensional representations of a cyclic group $\mathbb{Z}_{n}$ of order $n$. Consider $\mathbb{Z}_{n}$ as being identified with the $n$-th roots of unity, so $\mathbb{Z}_{n} \subset \mathbb{C}$. The underlying vector space of the representation $t^{k}$ is $\mathbb{C}$, and under the action $(g, v)$ is mapped to $g^{k} v$. For any cyclic group $G$ of order $n$ the complex representation ring $R(G)$ is isomorphic to $\mathbb{Z}[t] /\left(t^{n}-1\right)$. Thus any complex representation can be written as a linear combination of the elements in $\left\{t^{k} \mid k=0, \ldots, n-1\right\}$.

Let $G$ be cyclic of order $n$, and let $V=\sum a_{k} t^{k}$ be a complex representation of $G$. For $g \in G$ such that the fixed point set $V^{g}=$ \{0\} Atiyah and Bott $[\mathbf{A B}]$ defined a complex number

$$
\nu(V)(g)=\prod\left(\frac{1+g^{k}}{1-g^{k}}\right)^{a_{k}} \in \mathbb{C}-\{0\}
$$


Note that $\nu$ carries sums to products; so we can define

$$
\nu(V-W)(g)=\nu(V)(g) / \nu(W)(g)
$$

for any two representations $V$ and $W$ of $G$ for which $\nu$ is defined. Suppose $V$ and $W$ are Smith equivalent representations of $\mathbb{Z}_{n}$ supported by an even-dimensional homotopy sphere $\Sigma$, i.e., $\Sigma^{G}=\{p, q\}$ and $T_{p} \Sigma=V, T_{q} \Sigma=W$. The Atiyah-Singer $G$ Signature Theorem, the Lefschetz Fixed Point Theorem, and Smith Theory imply

Condition. 2.1. (1) $\operatorname{Sign}\left(G, \Sigma^{P}\right)=0$ for $P \in \mathscr{P}(G)$. In particular, $\nu\left(V^{P}-W^{P}\right)(g)= \pm 1$ if $V^{\langle P, g\rangle}=W^{\langle P, g\rangle}=0$. Here $\langle P, g\rangle$ denotes the group generated by $P$ and $g \in G$.

(2) The Euler characteristic $\chi\left(\Sigma^{K}\right)=2$ for all subgroups $K \subset G$.

(3) $\operatorname{Res}_{P} V \cong \operatorname{Res}_{P} W$ for each $P \in \mathscr{P}(G)$ of odd prime power order.

An easy computation shows that $\nu(V)(g)= \pm \nu\left(V^{\prime}\right)(g)$ whenever $V$ and $V^{\prime}$ are isomorphic as real representations. Let $U$ and $U^{\prime}$ be real representations of $G$ such that $U^{g}=U^{\prime g}=0$. We write

$$
\nu(U)(g)= \pm \nu\left(U^{\prime}\right)(g) \text { or } \quad \nu\left(U-U^{\prime}\right)(g)= \pm 1
$$

if $U$ and $U^{\prime}$ are realifications of complex representations $V$ and $V^{\prime}$ such that $\nu(V)(g)= \pm \nu\left(V^{\prime}\right)(g)$. This explains our notation in 2.1(1).

In order to find non-isomorphic Smith equivalent representations we have to start out with two non-isomorphic representations which satisfy $2.1(1)$ and 2.1(3). Actually we will make some additional assumptions in 2.2 which will allow us to carry out the construction of the homotopy sphere which supports the Smith equivalent representations.

From now on, unless specifically stated otherwise, $G$ denotes a cyclic group of order $2 n$ where $n$ is odd and $H$ is the index two subgroup of $G$. Sometimes $H$ just denotes an odd order cyclic group.

Consider pairs $(A, B)$ of real representations of $H$ satisfying

Condition. 2.2. (1) $A^{h}=B^{h}=0$ for each $h \in H$ which generates a subgroup of prime power index in $H$.

(2) $\operatorname{dim} A^{K}=\operatorname{dim} B^{K}$ whenever $|H / K|$ is divisible by at most 3 distinct primes.

(3) $\operatorname{Res}_{P} A \cong \operatorname{Res}_{P} B$ whenever $P \in \mathscr{P}(H)$.

(4) $\nu\left(A^{P}-B^{P}\right)(h)= \pm 1$ when ever $P \in \mathscr{P}$ and $h \in H$ generate a subgroup of prime power index in $H$. 
Observe that 2.2 (3) and some of the conditions in 2.2 (4) are necessary if we have Smith equivalent representations $V$ and $W$ of $G$ which restrict to the representations $A$ and $B$ of $H$. For this compare the reference to [Su2] in the introduction. There are two references which guarantee the existence of groups $H$ which have nonisomorphic representations satisfying all conditions in 2.2.

THEOREM 23 ([DP2, Corollary C]). There are odd order cyclic groups which have non-isomorphic real representations satisfying Condition 2.2. If $\mathbb{Z}_{m}$ has non-isomorphic representations which satisfy Condition 2.2 and $m$ divides an odd integer $m^{\prime}$, then $\mathbb{Z}_{m^{\prime}}$ has also nonisomorphic representations which satisfy Condition 2.2.

The groups in this theorem are rather large, and the representations cannot be given explicitly. The next reference improves on this result. In [DW] the reader can also find examples of non-isomorphic representations which satisfy the conditions in 2.2 for groups as in the next theorem.

THEOREM 2.4 ([DW, Theorem A]). Let $m=p_{1}, \ldots, p_{k}$ be a squarefree odd integer such that $p_{1}$ is congruent to 5 modulo 8 , the Legendre symbols $\left[\frac{p_{j}}{p_{1}}\right]$ are 1 for $j \geq 2$, and $k \geq 4$. Then the group $\mathbb{Z}_{m}$ has non-isomorphic representations which satisfy 2.2 .

3. One fixed point actions on manifolds. Our starting point is the construction of cyclic actions on surfaces. We shall use the following conventions. A real representation $U$ of $L$ is $L$ oriented if $U^{K}$ is an oriented vector space for all $K \subset L$. A smooth $L$ manifold $X$ is $L$ oriented if for all $K \subset L$ each component of $X^{K}$ is oriented. Since a complex vector space understood as a real vector space has a canonical orientation, the realification of a complex representation of $L$ is canonically $L$ oriented. If $X$ is an $L$ oriented manifold, then $T_{x} X$ is an $L_{x}$ oriented representation. Here $L_{x}=\{g \in L \mid g x=x\}$ is the isotropy group at $x$. Let $U$ be a representation. A product bundle $X \times U$ over $X$ is denoted by $\underline{U}$ when the base space $X$ is understood from context.

Let $L=\mathbb{Z}_{m}$ be a cyclic group of order $m$, and $t^{k}$ the complex representation of $\mathbb{Z}_{m}$ from the previous section, $(k, m)=1$. Let $A_{+}$ and $A_{-}$be finite $\mathbb{Z}_{m}$ sets of the same cardinality, $\left|A_{+}\right|=\left|A_{-}\right|$. 
Proposition 3.1. (See [DP2, 3.15].) There exists an oriented closed surface $S$ with smooth orientation preserving action of $\mathbb{Z}_{m}$ such that

(1) $S^{K}=A_{+}^{K} \cup A_{-}^{K}$ for all proper subgroups $K$ of $L$.

(2) The tangent bundle TS is stably isomorphic to the product bundle $S \times t^{k}$.

(3) $T_{x} S=\operatorname{Res}_{K} t^{ \pm k}$ if $x \in A_{ \pm}^{K}$ and $K \subset L$. (Observe that $T_{x} S$ and $\operatorname{Res} t^{ \pm k}$ are oriented representations, and the isomorphism is assumed to preserve orientations.)

The problem in the application of Proposition 3.1 is the choice of the sets $A_{+}$and $A_{-}$. To indicate our choice we need some more notation. Let $t^{k}=\psi$ be an irreducible representation of $G=\mathbb{Z}_{2 n}$. We suppose that $2 n /(k, 2 n)$ is divisible by at least two odd primes. We assign to it the group $L(\psi)$ which acts effectively on $\psi$. It is obtained as follows. Let $\operatorname{ker}(\psi)$ be the kernel of the homomorphism $\psi_{0}: G \rightarrow U(1)$ associated with $\psi$. This kernel is also the isotropy group of any non-zero vector in $\psi$. Then $L(\psi)=G / \operatorname{ker}(\psi)$. Let $m(\psi)$ be the order of $L(\psi)$. Then $m(\psi)=2 n /(k, 2 n)$. Observe that $(m(\psi), k)=1$. Now $A_{+}$and $A_{-}$are chosen as $L(\psi)=\mathbb{Z}_{m(\psi)}$ sets. The choice will depend on $m(\psi)$ only.

Choice 3.2. (1) If $m(\psi)$ is odd we decompose $m(\psi)$ as a product $m(\psi)=r(\psi) s(\psi)$ such that $(r(\psi), s(\psi))=1$. If $m(\psi)$ is divisible by four primes we suppose that $r(\psi)$ and $s(\psi)$ are divisible by at least two primes. Choose $a(\psi)$ and $b(\psi)$ as natural numbers such that $a(\psi) r(\psi)+b(\psi) s(\psi)+1 \equiv 0(\bmod m(\psi))$.

(2) If $m(\psi)$ is even, we set $m^{\prime}(\psi)=m(\psi) / 2$. As in (1), we decompose $m^{\prime}(\psi)$ as $r(\psi) s(\psi)$ such that $(r(\psi), s(\psi))=1$. Then we choose natural numbers $a(\psi)$ and $b(\psi)$ such that $a(\psi) r(\psi)+b(\psi) s(\psi)+1 \equiv$ $0(\bmod m(\psi))$.

In case (1) we set

$$
A_{+}(\psi)=b(\psi) \cdot\left[\mathbb{Z}_{m(\psi)} / \mathbb{Z}_{r(\psi)}\right] \sqcup a(\psi) \cdot\left[\mathbb{Z}_{m(\psi)} / \mathbb{Z}_{s(\psi)}\right] \sqcup\left[\mathbb{Z}_{m(\psi)} / \mathbb{Z}_{m(\psi)}\right]
$$

In case (2) we set

$A_{+}(\psi)=b(\psi) \cdot\left[\mathbb{Z}_{m(\psi)} / \mathbb{Z}_{2 r(\psi)}\right] \sqcup a(\psi) \cdot\left[\mathbb{Z}_{m(\psi)} / \mathbb{Z}_{2 s(\psi)}\right] \sqcup\left[\mathbb{Z}_{m(\psi)} / \mathbb{Z}_{m(\psi)}\right]$.

In either case $\left|A_{+}(\psi)\right| \equiv 0(\bmod m(\psi))$ such that we can choose $A_{-}(\psi)$ as free $\mathbb{Z}_{m(\psi)}$ set with the same cardinality as $A_{+}(\psi)$.

In the second step we assign to each irreducible complex representation $\psi=t^{k}$, for which $m(\psi)$ is divisible by at least two primes, a 
surface $X(\psi)$ with $G$ action. First we use Lemma 3.1 to define an $L(\psi)$ action on a surface which we call $X^{\prime}(\psi)$. Reduction modulo $m(\psi)$ defines a homomorphism $G \rightarrow L(\psi)$ and this induces a $G$ action on $X^{\prime}(\psi)$. The surface with this induced $G$ action is denoted by $X(\psi)$.

We describe the properties of these surfaces $X(\psi)$, which are almost identical with those listed in [DP2, Corollary 3.5]. As before we suppose that $m(\psi)$ is divisible by at least two odd primes.

Lemma 3.3. Let $X(\psi)$ be as above, and $G(\psi)=\operatorname{ker}(\psi)$.

(1) $\operatorname{Res}_{G(\psi)} X(\psi)$ (with its trivial $G(\psi)$ action) is a $G(\psi)$ oriented boundary.

(2) There is a representation $A$ of $G$ such that $T X(\psi) \oplus \underline{A}=\underline{\psi} \oplus \underline{A}$. For all subgroups $K$ of $G$ such that $|G / K|=1,2$, an odd prime, or twice an odd prime

(3) $X(\psi)^{K}$ is a finite set.

(4) $\left|X(\psi)^{G}\right|=1$, and $\left|X(\psi)^{K}\right|=1$ whenever $|G / G(\psi)|=|L(\psi)|$ is divisible by at least four distinct odd primes.

(5) $\left|X\left(\psi^{\prime}\right)^{K}\right|=\left|X\left(\psi^{\prime \prime}\right)^{K}\right|$ whenever $G\left(\psi^{\prime}\right)=G\left(\psi^{\prime \prime}\right)$.

(6) If $G(\psi)=1$ or $G(\psi) \supset \mathbb{Z}_{2}$ then every isotropy group of $X(\psi)$ is 1 , or it contains $\mathbb{Z}_{2}$. Thus $G$ acts freely on $X(\psi)-X(\psi)^{\mathbb{Z}_{2}}$.

Proof. Only (6) does not occur in [DP2]. It is an immediate consequence of our choice of $A_{+}$and $A_{-}$.

Let $U$ be a complex representation of a cyclic group $G$ such that $U^{G}=0$ and $U^{K}=0$ whenever $|G / K|=1$, an odd prime, or twice an odd prime. Then $U$ is a direct sum of non-trivial irreducible representations, $U=\sum a_{\psi}(U) \psi$. For each irreducible representation $\psi$ for which $a_{\psi}(U) \neq 0$ the assumption on $m(\psi)$ is satisfied and $X(\psi)$ is defined. We now define a $G$ oriented manifold

$$
X(U)=\prod X(\psi)^{a_{\psi}(U)} .
$$

The exponent $a_{\psi}$ indicates an $a_{\psi}$-fold cartesian product of $X(\psi)$ with itself. Next we study the properties of this manifold. They are derived from Lemma 3.3. These properties are exactly those in [DP2, 3.6-3.11], and the proof is unchanged as well.

COROLlARY 3.5. Let $U$ be a complex representation of $G$ satisfying $U^{K}=0$ if and only if $|G / K|=1,2$, an odd prime, or twice an odd prime. Let $X(U)$ be the $G$ oriented manifold in 3.4. Then 
(1) There is a representation $C$ of $G$ such that $T X(U) \oplus \underline{C}$ and $\underline{U} \oplus \underline{C}$ are isomorphic $G$ vector bundles.

(2) $\operatorname{dim} X(U)^{K}=0$ if and only if $|G / K|=1,2$, an odd prime, or twice an odd prime.

(3) If $|G / K| \neq 1,2$, not an odd prime, and not twice an odd prime $\operatorname{Res}_{K} X(U)$ bounds as an oriented $K$ manifold.

(4) $\chi\left(X(U)^{G}\right)=1$ and $\chi\left(X(U)^{K}\right)$ is even whenever $|G / K|$ is not 1,2 , an odd prime, and not twice an odd prime.

(5) Suppose $|G| K \mid=1,2$, an odd prime, or twice an odd prime. Then $X(U)^{K}$ is a finite set and if $y \in X(U)^{K}$, then $T_{y} X(U)=\operatorname{Res}_{K} U$ as $K$ oriented real representation. The cardinality of $X(U)^{K}$ depends only on $\left\{\left(K, \operatorname{dim} U^{K}\right) \mid K \subset G\right.$ and $|G / K|$ is divisible by at most three distinct odd primes $\}$.

To obtain Theorem A we will start with a collection $\mathscr{S}$ of complex representations of $G=H \times \mathbb{Z}_{2}$ where $H$ is an odd order cyclic group. If $U$ is a representation of $G$ we denote its $\mathbb{Z}_{2}$ fixed point set $U^{\mathbb{Z}_{2}}$ by $U_{2}$ which we also consider as representation of $H$. The complement is denoted by $U_{f}$, so $U=U_{2} \oplus U_{f}$. The representations in $\mathscr{S}$ are assumed to satisfy

Condition. 3.6. (1) If $U \in \mathscr{S}$ and $K \subset G$, then $U^{K}=0$ if and only if $|G / K|=1,2$, an odd prime, or twice an odd prime.

(2) If $K \in \operatorname{Iso}(U)$ then $K=1$ or $K \supseteq \mathbb{Z}_{2}$.

Each pair $(D, E)$ of representations in $\mathscr{S}$ satisfies

(3) $\operatorname{dim} D^{K}=\operatorname{dim} E^{K}$ if $|G / K|$ is divisible by at most three distinct odd primes. Furthermore $\operatorname{dim} D=\operatorname{dim} E$ and $\operatorname{dim} D_{2}=\operatorname{dim} E_{2}$.

(4) $\nu\left(D_{2}^{P}-E_{2}^{P}\right)(g)=1$ whenever $P \in \mathscr{P}(H)$ and $g \in H$ generates a subgroup of prime power index in $H$.

(5) $D_{f}=E_{f}$ as representations of $G$.

Note on Condition 3.6 (2). This condition expresses that if $\psi$ is an irreducible summand of $U$ then $\psi$ is a summand of $U_{2}$ or $G$ acts freely on $\psi-\{0\}$. Hence $G$ acts freely on $U-U_{2}$.

We list the essential properties of the manifolds $X(U)$ obtained from a collection of representations $\mathscr{S}$ as in 3.6 when $U \in \mathscr{S}$. It should be compared with [DP2, Theorem D on page 289]. We denote $X(U)^{\mathbb{Z}}{ }_{2}$ by $X_{2}(U)$ and consider it as an oriented $H$ manifold.

THEOREM 3.7. Let $G=\mathbb{Z}_{2 n}$ ( $n$ odd) and $\mathscr{S}$ a collection of representations of $G$ which satisfy $3.6(1)-(4)$. There is a collection of 
closed $G$ oriented manifolds $\{X(U) \mid U \in \mathscr{S}\}$ such that

(1) $X(U)^{G}=x$ consists of exactly one point and $T_{x} X(U)=U$ as $G$ oriented real representation.

(2) There is a representation $C$ of $G$ such that $T X(U) \oplus \underline{C}$ and $\underline{U} \oplus \underline{C}$ are isomorphic $G$ vector bundles.

(3) $G$ acts freely on $X(U)-X_{2}(U)$.

(4) $\chi\left(X(D)^{K}\right) \equiv \chi\left(X(E)^{K}\right)(\bmod 2)$ for all $K \subset G$ and $D, E \in \mathscr{S}$.

(5) $\operatorname{Sign}\left(H, X_{2}\left(D^{P}\right)\right)=\operatorname{Sign}\left(H, X_{2}\left(E^{P}\right)\right)$ for all $P \in \mathscr{P}(H)$ and for all $D, E \in \mathscr{S}$.

(6) $\operatorname{Sign}(G, X(D))=\operatorname{Sign}(G, X(E))$ for all $D, E \in \mathscr{S}$.

Proof. The first two properties (1) and (2) are repetitions of 3.5 (1) and (2). Property (3) follows from 3.3 (6) and 3.6 (2). See also above note on 3.6 (2). To see (4) observe that $X(U)=X\left(U_{2}\right) \times X\left(U_{f}\right)$. It follows from [DP2, Theorem D (ii) page 289] that

$$
\chi\left(X\left(D_{2}\right)^{K}\right) \equiv \chi\left(X\left(E_{2}\right)^{K}\right) \quad(\bmod 2)
$$

for all $K \subset H \subset G$. Furthermore

$$
\chi\left(X\left(D_{f}\right)^{K}\right) \equiv \chi\left(X\left(E_{f}\right)^{K}\right) \quad(\bmod 2)
$$

because of $3.6(2)$ and (5) and $3.5(4)-(5)$. These two congruences imply our claim (4).

To see (5), let $S_{D}$ denote the singular set of $X\left(D_{f}\right)$. It consists of the non-free orbits in $X\left(D_{f}\right)$, which is a finite set. Thus

$$
X_{2}(D)=S_{D} \times X\left(D_{2}\right) \text { and } X_{2}(E)=S_{E} \times X\left(E_{2}\right)
$$

It has been verified in [DP2, Theorem D (iii)] that $X\left(D_{2}\right)$ and $X\left(E_{2}\right)$ have the property stated in (5). By construction $S_{D}=S_{E}$. From this it follows trivially that (5) holds.

To see (6) one proceeds in exactly the same way as in the proof of [DP2, Theorem D (iii)].

3.1. Addendum. There is another property which we may impose for the surface $S$ in Lemma 3.3. This has an implication for the conclusion in Corollary 3.5 which is elementary, and which we leave to the reader. We state them.

Additional Properties.

3.3 (6) We may choose the surface $X(\psi)$, the zero cobordism $W$, and the stable $G$ trivialization of the tangent bundle $T X(\psi)$ such 
that it extends (after restriction to a $G(\psi)$ trivialization) to a stable $G(\psi)$ trivialization of $T W$.

3.5 (6) If we assume a choice as in 3.3 (6), then there is a stable $K$ trivialization of the tangent bundle of the zero cobordism of $\operatorname{Res}_{K} X(U)$ which extends the stable trivialization of $T X(U)$ in 3.5 (1), restricted to a $K$ action.

Proof of $3.3(6)$. Consider the surface $X^{\prime}(\psi)$ with smooth $L(\psi)$ action which give rise to the surfaces $X(\psi)$. Abbreviate $X^{\prime}(\psi)$ by $X^{\prime}$ and $L(\psi)$ by $L$. Let $L_{2}$ be the 2-Sylow subgroup of $L$. Let $W^{\prime}$ be an oriented manifold which bounds $X^{\prime}$. We may suppose that $T W^{\prime}$ is trivial. Define

$$
X^{\prime \prime}=X^{\prime} \sqcup L \times_{L_{2}} X^{\prime} \quad \text { and } \quad W^{\prime \prime}=W^{\prime} \sqcup L \times_{L_{2}} W^{\prime} .
$$

Then $W^{\prime \prime}$ bounds $X^{\prime \prime}$. The stable $L$ trivialization of $T X^{\prime}$ induces one on $T X^{\prime \prime}$, and we show that this one extends to a non-equivariant stable trivialization of the tangent bundle of some manifold which bounds $X^{\prime \prime}$.

There may be an obstruction for extending the stable trivialization of $T X^{\prime}$ to one of $T W^{\prime}$ (after forgetting the action), and such obstructions lie in $\pi_{j}(S O)$ for $0 \leq j \leq 2$. Among these $\pi_{1}(S O)=\mathbb{Z}_{2}$, and the other groups vanish. After some zero-dimensional surgeries we may assume that $W^{\prime \prime}$ is connected, and in this case a possible initial obstruction for the extension of the bundle trivialization has been multiplied by $1+\left|L / L_{2}\right| \equiv 0(\bmod 2)$. Thus for this modified $W^{\prime \prime}$ and our original $X^{\prime \prime}$ with its stable trivialization of its tangent bundle the obstruction vanishes. So the trivialization of $T X^{\prime \prime}$ (without group action) extends to one of $T W^{\prime \prime}$.

Again we use the projection $G \rightarrow L$ to induce a $G$ action on $X^{\prime \prime}$ and the trivial $G(\psi)$ action on $W^{\prime \prime}$. With these induced actions the manifolds are denoted by $X(\psi)$ and $W$. They have all of the properties listed in $3.3(1)-(6)$.

4. Proof of Theorem A. Throughout the section we will use $k=1$, which means that $G=H \times \mathbb{Z}_{2}$. The case $k \geq 1$ causes only some additional notational effort, but otherwise it is identical.

Sketch of Proof of Theorem A. We start out with a sufficiently large collection $\mathscr{S}$ of representations of $G=H \times \mathbb{Z}_{2}$ which satisfies Condition 4.3. Using Theorem 3.7 we associate to each pair $(V, W)$ of representations in $\mathscr{S}$ a smooth $G$ manifold $X(V, W)$ with exactly 
two fixed points and tangent representations $V$ and $W$. The choices will be such that $X^{s}=X^{\mathbb{Z}_{2}}$, where $X^{s}$ denotes the set of non-free orbits of $X$. It follows from [DP2] that for a certain subset $\mathscr{T}$ of $\mathscr{S}$ and $V, W \in \mathscr{T}$ that $X(V, W)$ is equivariantly cobordant relative to the fixed point set to a $G$ manifold $Y(V, W)$ such that $Y^{s}=Y^{\mathbb{Z}_{2}}$ is a homotopy sphere. The cardinality of $\mathscr{T}$ is at least a fraction of the one of $\mathscr{S}$. The surgery obstruction which tells whether $Y(V, W)$ is $G$ cobordant to a homotopy sphere lies in $L_{0}^{h}(\mathbb{Z}[G], 1)$. In fact, it lies in a finite subgroup $\mathscr{I}$ due to the signature computation in 3.7 (6). We choose $|\mathscr{S}|$ such that $|\mathscr{T}| \geq|\mathscr{F}|$. Based on the additivity of the surgery obstruction and the pigeon hole principle we find $V$ and $W$ such that the obstruction vanishes for $Y(V, W)$, which is then $G$ cobordant to a homotopy sphere.

Before we can prove Theorem A we need two technical definitions.

Definition 4.1. A $G$ manifold $X$ is defined to be stable if for each $x \in X$ and $K=G_{x}$, the multiplicity $m_{\chi}\left(T_{x} X\right)$ of each nontrivial irreducible representation $\chi$ in $T_{x} X=V$ is either zero or $d_{\chi} m_{\chi}(V) \geq \operatorname{dim}_{\mathbb{R}} V^{K}$. Here $d_{\chi}=\operatorname{dim}_{\mathbb{R}} D_{\chi}$, and $D_{\chi}$ is the algebra of real $K$ endomorphisms of $\chi$.

Definition 4.2. A representation $V$ of $G$ satisfies the gap hypothesis if for any two representations $L \subset K \subset G$ either $V^{K}=V^{L}$ or $2 \operatorname{dim} V^{K}+1<\operatorname{dim} V^{L}$. A smooth $G$ manifold $X$ satisfies the gap hypothesis if $T_{X} X$ satisfies the gap hypothesis with respect to the induced $G_{x}$ action for every $x \in X$.

To prove Theorem A we proceed as in $\S 7$ of [DP2]. We consider a collection $\mathscr{S}$ of complex representations of $G=H \times \mathbb{Z}_{2}$ and a representation $U$. Together they are assumed to satisfy

Condition 4.3. (1) $U$ is stable, satisfies the gap hypothesis, and the isotropy groups of $U$ are all the subgroups of $G$ which contain $\mathbb{Z}_{2}$ and the trivial group. If $\operatorname{dim} U^{K} \neq 0$, then $\operatorname{dim} U^{K} \geq 6$. Furthermore $\operatorname{dim} U^{K} \equiv 0(\bmod 4)$ for all $K \subset G$.

(2) $D \in \mathscr{S}$ is stable, satisfies the gap hypothesis, and $K$ is an isotropy group of $D$ if and only if $K=G, K=1$, or $\mathbb{Z}_{2} \subset K$ and $G / K \notin \mathscr{P}$. If $\operatorname{dim} D^{K} \neq 0$ then $\operatorname{dim} D^{K} \geq 6$. Furthermore $\operatorname{dim} U^{K} \equiv 0(\bmod 4)$ for all $K \subset G$. 
(3) With the induced $H$ action we have $\operatorname{Res}_{P} U^{\mathbb{Z}_{2}}=\operatorname{Res}_{P} D^{\mathbb{Z}_{2}}$ for all $D \in \mathscr{S}$ and for all $P \in \mathscr{P}(H)$.

(4) Each pair $(D, E) \in \mathscr{S}$ satisfies Condition 3.6 (3)-(5).

LEMMA 4.4. Given a pair of non-isomorphic representations $(A, B)$ of real representations of $H$ which satisfies 2.2 and any number $N$, there exists a collection $\mathscr{S}$ of non-isomorphic representations of $G$ which satisfies Condition 4.3, and the cardinality of $\mathscr{S}$ is greater than or equal to $N$.

Proof. Consider the representations $R_{j}=(N-j) A \oplus j B$ of $H$. Any pair of them satisfies Condition 2.2, and so do their complexifications $\widetilde{R}_{j}$. Via the projection $G \rightarrow H$ they are considered as representations of $G$. It is explained in [DP2] how to find a representation $U_{0}$ and a representation $U$ of $G$ such that $\mathscr{S}=\left\{\widetilde{R}_{j} \oplus U_{0} \mid 0 \leq j \leq N\right\}$ and $U$ satisfy Condition 4.3. More precisely, first we do this for the $G$ (or $H$ ) representations $\left\{j A^{\mathbb{Z}_{2} \oplus} \oplus(N-j) B^{\mathbb{Z}_{2}}\right\}$ according to [DP2], and then we add the representation $t^{1}$ of $G$ sufficiently often to each of them. This produces the desired set $\mathscr{S}$ and the representation $U$.

Our next result uses the notion of a special Smith framed manifold. Both are technical concepts which we do not want to review. A Smith framing provides bundle data used in the process of equivariant surgery. It was introduced in [PR1], and it was also summarized in [DP2, §5]. The word special refers to some properties listed in [DP2, Definition 5.14]. Both will be only of minor relevance to our argument.

LEMMA 4.5. Let $U$ be a representation of $G$ and $\mathscr{S}$ a collection of representations as in 4.3. For any pair $(V, W)$ of elements in $\mathscr{S}$ there is a $U$ Smith framed $G$ manifold $X=X(V, W)$ with exactly two fixed points $x$ and $y$, and as $G$ oriented representations $T_{X} X=V$ and $T_{y} X=-W$. In addition $X^{\mathbb{Z}_{2}}$ is a special $H$ manifold, and $\left(X^{\mathbb{Z}_{2}}\right)^{P}$ is a simply connected mod $p$ homology sphere for each nontrivial $P \in \mathscr{P}(H)$ of $p$ power order.

Adaptation of proof. This is basically Lemma 7.3 in [DP2]. An initial approximation of $X(V, W)$ is given as $X(V) \sqcup-X(W) \sqcup Z$, where $X(V)$ and $X(W)$ are as in 3.4 and $Z$ is a zero cobordant $G$ manifold constructed from $U$ used to adjust Euler characteristics (see [DP2, page 303]). Equivariant surgery provides a cobordism between 
this initial manifold and the desired $X(V, W)$. These surgeries are performed in our setting as surgeries on the $\mathbb{Z}_{2}$ fixed point set of a $G$ manifold on which the odd order group $H$ acts. In [DP2] one utilizes the properties of the one fixed point manifolds summarized in Theorem $\mathrm{D}$ of the reference. In our setting the required properties of the one fixed point manifolds are listed in Theorem 3.7.

To the manifolds $X(V, W)$ of the lemma one may assign an obstruction $\sigma(V, W)$. It has the following

Properties 4.6 (Compare [DP2, Lemma 5.24]). (1) $\sigma(V, W)$ lies in a finite group whose order depends only on $H$.

(2) If $\sigma(V, W)=0$ then $X(V, W)$ is $U$ Smith framed $G$ cobordant to a manifold $Y(V, W)$ such that $Y(V, W)^{\mathbb{Z}_{2}}$ has all of the properties listed in 4.5 , but in addition $Y(V, W)^{\mathbb{Z}_{2}}$ is a homotopy sphere.

(3) $\sigma(V, W)$ is additive under connected sum at a fixed point, that is $\sigma(V, W)+\sigma\left(W, W^{\prime}\right)=\sigma\left(V, W^{\prime}\right)$ for all $V, W, W^{\prime} \in \mathscr{S}$.

(4) $\sigma(V, W)=-\sigma(W, V)$ for $V, W \in \mathscr{S}$.

Note to 4.6 (3). The possibility to give a $U$ Smith framing to a fixed point connected sum $X(V, W) \# X\left(W, W^{\prime}\right)$ at the fixed point with tangent representation $W$ depends on a compatible choice of $U$ Smith framing for $X(V, W)$ and $X\left(W, W^{\prime}\right)$ provided in [DP2, $\left.7.3^{\prime}\right]$.

Our next result is a refinement of [DP2, Theorem 7.4].

THEOREM 4.7. Given a representation $U$ of $G$ and a collection $\mathscr{S}$ of representations of $G$ as in 4.3 with cardinality $N$. There exists a natural number $N_{0}$ and a collection $\left\{X\left(W_{i}, W_{j}\right)\right\}$ for $1 \leq i, j \leq$ $N / N_{0}-1$ such that

(1) $X\left(W_{i}, W_{j}\right)$ is a smooth $G$ manifold with exactly two fixed points at which the $G$ oriented tangent representations are $W_{i}$ and $-W_{j}$ for $W_{i}, W_{j} \in \mathscr{S}$.

(2) $X\left(W_{i}, W_{j}\right)^{\mathbb{Z}_{2}}$ is a homotopy sphere and $G$ acts freely on $X-$ $X^{\mathbb{Z}_{2}}$.

(3) $X\left(W_{i}, W_{j}\right)$ is $U$ Smith framed.

(4) $W_{i} \neq W_{j}$ if $i \neq j$.

(5) $X\left(W_{i}, W_{j}\right)$ is stable and satisfies the gap hypothesis.

(6) $\operatorname{Sign}\left(G, X\left(W_{i}, W_{j}\right)\right)=0$. 
Proof. Suppose $N_{0}$ is larger than the order of the obstruction group in 4.6 (1). Choose $V \in \mathscr{S}$ and consider the set $\left\{X\left(V, W_{j}\right) \mid W_{j} \neq\right.$ $V$ and $\left.W_{j} \in \mathscr{S}\right\}$. For at least $N / N_{0}$ of them $\sigma\left(V, W_{i}\right)=\sigma\left(V, W_{j}\right)$ for $i \neq j$. We now use $X\left(W_{i}, V\right) \# X\left(V, W_{j}\right)$ as $X\left(W_{i}, W_{j}\right)$. According to $4.6(3)$ and (4) $\sigma\left(W_{i}, W_{j}\right)=0$. It follows from 4.6 (2) that $X\left(W_{i}, W_{j}\right)$ is $U$ Smith framed $G$ cobordant to a manifold having properties (1)-(5) of our claims. By construction $X(V, W)$ is equivariantly cobordant to $X(V) \sqcup-X(W)$, and it follows from 3.7 (6) that $\operatorname{Sign}(G, X(V, W))=0$ for all $V, W \in \mathscr{S}$. This implies the last part of our claim.

Our next result is the key for altering manifolds as in 4.7 once more such that $X\left(W_{i}, W_{j}\right)$ is a homotopy sphere.

THEOREM 4.8. Let $G=H \times \mathbb{Z}_{2}$ and $X$ a smooth $G$ manifold such that

(1) $X$ is Smith framed of dimension congruent to 0 modulo 4.

(2) $X$ is stable and satisfies the gap hypothesis.

(3) $X^{\mathbb{Z}}{ }_{2}$ is a homotopy sphere.

(4) $X$ has a fixed point and $G$ acts freely on $X-X^{\mathbb{Z}_{2}}$.

(5) $\operatorname{Sign}(G, X)=0$.

There exists an obstruction $\sigma(X)$ in a finite group, and if $\sigma(X)=0$ then $X$ is Smith framed $G$ cobordant to a homotopy sphere relative to the $\mathbb{Z}_{2}$ fixed point set.

Proof. Surgery obstructions are usually assigned to normal maps. Let $x$ be a fixed point of $X$ with tangent representation $\Omega$. There is a standard map $f: X \rightarrow Y=S(\Omega \oplus \mathbb{R})$ of degree 1 . which collapses the complement of $\Omega$ embedded a neighbourhood of $x$ to a point. The Smith framing on $X$ provides bundle data $b$ which allow us to apply equivariant surgery. The data $(X, f, b)$ denote the resulting normal map. For the definition of a normal map as it applies to our present situation see [DP2, Definition 5.15] or [PR1].

After some equivariant surgeries on $X$ in the free part and below the middle dimension we may suppose that $f$ is $2 k$-connected, here the dimension of $X$ is assumed to be $4 k$. We shall show later in the proof that

$$
K_{2 k}(X)=\operatorname{ker}\left(f_{*}: H_{2 k}(X) \rightarrow H_{2 k}(Y)\right)
$$

is a stably free $\mathbb{Z}[G]$ module. Together with the intersection form $\lambda$ and the self-intersection form $\mu$ (see [DR]) these data define a class $\sigma(f, b)=\left[K_{2 k}(X), \lambda, \mu\right] \in L_{0}^{h}(\mathbb{Z}[G], w)$. Here $w: G \rightarrow\{ \pm 1\}$ is 
a homomorphism and $w(g)=1$ if and only if $g$ acts orientation preservingly on $X$.

It is discussed in [DR] that $\sigma(f, b)$ is the surgery obstruction of $(X, f, b)$. In particular, if $\sigma(f, b)=0$ then $(X, f, b)$ is $U$ Smith framed $G$ normally cobordant to a normal map $f^{\prime}: \Sigma \rightarrow Y$ such that $f^{\prime}$ is a homotopy equivalence. This cobordism is relative to all non-free orbits. Due to assumption (5) of the theorem $\sigma(f, b)$ lies in a finite subgroup of $L_{0}^{h}(\mathbb{Z}[G], w)$, the kernel of the signature homomorphisms to the representation ring, Sign: $L_{0}^{h}(\mathbb{Z}[G], w) \rightarrow \mathbb{R}(G)$. From its definition it is clear that $\sigma(f, b)$ depends only on $X$ (and possibly its Smith framing) but not on $f$. So we denote it by $\sigma(X)$. This completes the proof of the theorem, except that we need to verify that $K_{2 k}(X)$ is a stably free $\mathbb{Z}[G]$ module.

Let $M$ denote the mapping cylinder. Let $X^{s}=\left\{x \in X \mid G_{x} \neq 1\right\}$ be the singular set, and $f^{s}$ the restriction of $f$ to the singular sets. There is a short exact sequence of $\mathbb{Z}[G]$ chain complexes:

$$
0 \rightarrow C_{*}\left(M_{f^{s}}, X^{s}\right) \rightarrow C_{*}\left(M_{f}, X\right) \rightarrow C_{*}\left(M_{f}, X \cup M_{f^{s}}\right) \rightarrow 0
$$

The homology of $C_{*}\left(M_{f^{s}}, X^{s}\right)$ vanishes because of assumption (3) and (4). Thus

$$
K_{2 k}(X) \cong H_{2 k+1}\left(C_{*}\left(M_{f}, X \cup M_{f^{s}}\right)\right)
$$

as $\mathbb{Z}[G]$ module. The homological assumptions on $C_{*}\left(M_{f}, X\right)$ implied by the fact that $f$ is $2 k$-connected also hold for $C_{*}\left(M_{f}, X \cup M_{f^{s}}\right)$. The latter complex is a free $\mathbb{Z}[G]$ chain complex. Thus it follows from [W, Lemma 2.3 (c)] that $K_{2 k}(X)$ is stably $\mathbb{Z}[G]$ free. In this reference one omits the discussion of the preferred stable basis. This completes our proof.

Proof of Theorem A. Choose $N \geq N_{0}\left(N_{1}+3\right)$ where $N_{1}$ is the order of the obstruction group referred to in Theorem 4.8, and $N_{0}$ is as in 4.7. Choose a representation $U$ of $G$ and a collection $\mathscr{S}$ (see 4.4) of cardinality at least $N$. Theorem 4.7 provides a subset $\mathscr{T} \subset \mathscr{S}$ of cardinality at least $N_{1}+2$ for which there exist manifolds $X\left(W_{i}, W_{j}\right)$ as in the conclusion of 4.7 whenever $W_{i}, W_{j} \in$ $\mathscr{T}$. According to Theorem 4.8 we assign to $X\left(W_{i}, W_{j}\right)$ the obstruction $\sigma\left(W_{i}, W_{j}\right)=\sigma\left(X\left(W_{i}, W_{j}\right)\right)$. Pick any $V \in \mathscr{T}$ and consider $\left\{X\left(V, W_{i}\right) \mid W_{i} \in \mathscr{T}\right.$ and $\left.V \neq W_{j}\right\}$. The cardinality of this set is larger than $N_{1}$, the order of the obstruction group in 4.8. Thus there exist representations $W_{i}, W_{j} \in \mathscr{T}$ such that $W_{i} \neq W_{j} \neq V \neq W_{i}$ but 
$\sigma\left(V, W_{i}\right)=\sigma\left(V, W_{j}\right)$. This obstruction $\sigma$ also has the properties 4.6 (3) and (4), and it follows that

$$
\sigma\left(X\left(V, W_{i}\right) \#-X\left(V, W_{j}\right)\right)=0 .
$$

The connected sum is taken at the fixed point with tangent representation $V$. According to Theorem $4.8 X\left(V, W_{i}\right) \#-X\left(V, W_{j}\right)$ is Smith framed $G$ cobordant to a homotopy sphere $\Sigma$ relative to the non-free orbits. By construction $\Sigma$ has exactly two fixed points $x$ and $y$ with tangent representations $W_{i}$ and $W_{j}$, and these are not isomorphic. If the elements of $\mathscr{S}$ are chosen as it is proposed in the proof of Theorem 4.4 then the difference of $W_{i}$ and $W_{j}$ is a non-zero multiple of the difference of $A$ and $B$.

5. Some elementary constructions with representations. The purpose of this section is to construct representations as they will be needed in the proof of Theorem B. The main result is Theorem 5.3 which we will use later, and the remaining part of this section is devoted to its proof.

First we discuss how to find representations which satisfy Condition 1.1. The condition is restated in the lemma.

LemMA 5.1. Suppose $G$ is an abelian group with at least three noncyclic Sylow subgroups. There exist non-isomorphic representations $A$ and $B$ of $G$ such that

(1) $A^{K}=B^{K}=0$ whenever $G / K$ is of prime power order.

(2) $\operatorname{Res}_{P} A \cong \operatorname{Res}_{P} B$ whenever $P \subset G$ is of prime power order.

(3) $\operatorname{dim} A^{K}=\operatorname{dim} B^{K}$ for all $K \subset G$.

Proof. Let $C$ be a cyclic factor of $G$ whose order is $p^{a} q^{b}$, where $a, b \geq 1$ and $p$ and $q$ are odd primes. Find $\alpha$ and $\beta, 1<\alpha, \beta<$ $p q-1$ such that

$$
\begin{array}{ll}
\alpha \equiv 1\left(\bmod p^{a}\right) & \beta \equiv 2\left(\bmod p^{a}\right) \\
\alpha \equiv 2\left(\bmod q^{b}\right) & \beta \equiv 1\left(\bmod q^{b}\right) .
\end{array}
$$

Such $\alpha$ and $\beta$ exist. Set

$$
A^{\prime}=t^{\alpha} \oplus t^{\beta} \quad \text { and } \quad B^{\prime}=t^{1} \oplus t^{2} .
$$

These are representations of $C$. Obviously $A^{\prime} \neq B^{\prime}$, but

$$
\operatorname{Res}_{\mathbb{Z}_{p^{a}}} A^{\prime} \cong \operatorname{Res}_{\mathbb{Z}_{p^{a}}} B^{\prime} \text { and } \operatorname{Res}_{\mathbb{Z}_{q^{b}}} A^{\prime} \cong \operatorname{Res}_{\mathbb{Z}_{q^{b}}} B^{\prime}
$$


Set $G=C \times L$ and let $L$ act trivially on $A^{\prime}$ and $B^{\prime}$. With this action of $G$ we call $A^{\prime}$ and $B^{\prime}$ now $A$ and $B$, and these representations obviously satisfy (1)-(3) in our claim.

As input in our proof of Theorem B we will need a complex representation $U$ of $G$ and a collection $\mathscr{S}$ of complex representations of $G$ such that

Condition 5.2. (1) $U$ is stable, satisfies the gap hypothesis, and the set of isotropy groups of $U$ consists of all subgroups of $G$. Also, $\operatorname{dim} U^{K}=0$ or $\geq 6$ for all $K \subset G$. (The word stable and gap hypothesis were defined in Definition 4.1 and 4.2).

(2) $V \in \mathscr{S}$ is stable, satisfies the gap hypothesis, and the set of isotropy groups of $V$ consists of $G$ and all subgroups $K \subset G$ such that $G / K$ is not of prime power order. Furthermore, $V^{G}=0$ and $\operatorname{dim} V^{K} \geq 6$ for the other isotropy groups of $V$.

(3) $\operatorname{Res}_{P} V \cong \operatorname{Res}_{P} U$ for all $P \subset G$ of prime power order and $V \in \mathscr{S}$.

(4) $\operatorname{dim} V^{K}=\operatorname{dim} W^{K}$ for all $K \subset G$ and all $V, W \in \mathscr{S}$.

Theorem 5.3. Given representations $A$ and $B$ of $G$ as in Condition 1.1 (or Lemma 5.1) and any integer $N$. There exist a complex representation $U$ and a collection $\mathscr{S}$ of complex representations of $G$ such that $U$ and $\mathscr{S}$ satisfy Condition 5.2 and such that $V-W$ is a non-zero multiple of $A-B$ whenever $V, W \in \mathscr{S}$.

The remaining part of this section is concerned with the proof of this theorem. To prove it we need a bit more technical preparation.

LemMA 5.4. Suppose $G$ is abelian with at least three non-cyclic Sylow subgroups and $K \subset G$ is a subgroup such that $G / K$ is of prime power order. There exist representations $A_{2}(K)$ and $U_{2}(K)$ of $G$ such that

(1) $A_{2}(K)^{J}=0$ whenever $G / J$ is of prime power order.

(2) $\operatorname{Res}_{P} A_{2}(K) \cong \operatorname{Res}_{P} U_{2}(K)$ whenever $P$ is of prime power order.

(3) The isotropy groups of $U_{2}(K)$ are $G, K$, and subgroups of $K$ (possibly not all of them).

Proof. Suppose $K \subset G$ such that $|G / K|=p^{d}$, where $d \geq 1$ and $p$ is a prime. 
Step 1. Consider first the case when $G / K$ is cyclic of order $p^{a}$, $a \geq 1$. Construct a cyclic group $L$ such that we have a commutative diagram

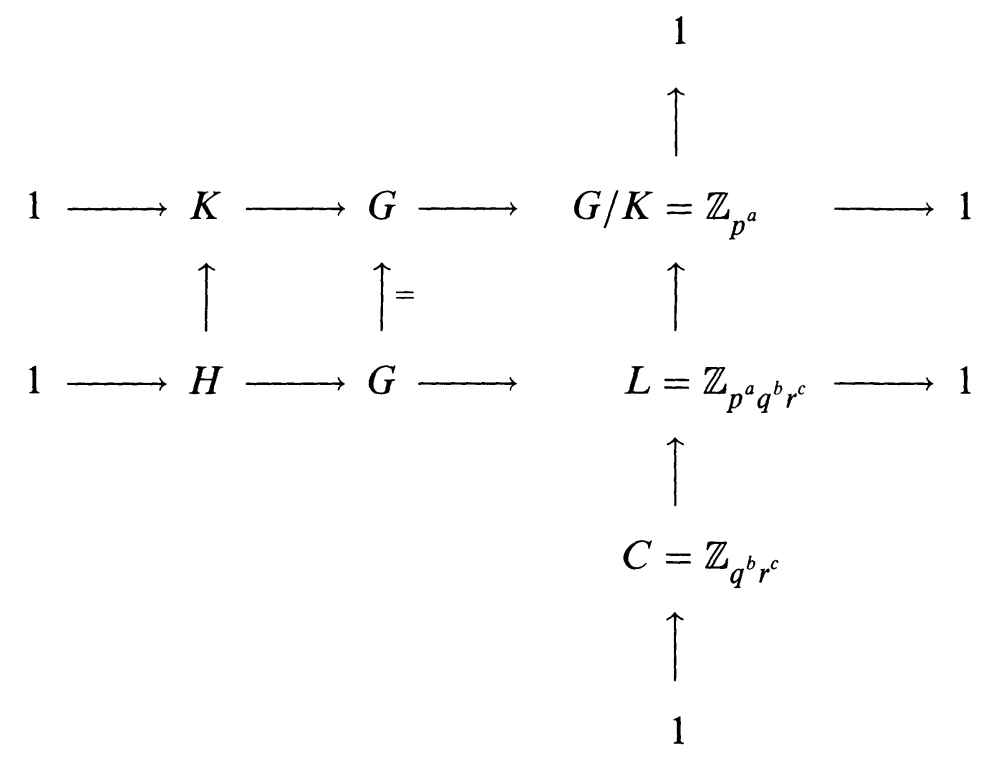

So $H$ is a subgroup of $K$ and $C$ is cyclic of order $q^{b} r^{c}$. Here $q$ and $r$ are distinct primes different from $p$, and $b, c \geq 1$. Let $\delta$ be an integer between 1 and $p^{a}$ which is prime to $p$. There are integers $\alpha, \beta$, and $\gamma, 1<\alpha, \beta, \gamma<p^{a} q^{b} r^{c}-1$, such that

$$
\begin{array}{lll}
\alpha \equiv \delta q^{b} r^{c}\left(\bmod p^{a}\right) & \beta \equiv 1\left(\bmod p^{a}\right) & \gamma \equiv 1\left(\bmod p^{a}\right) \\
\alpha \equiv 1\left(\bmod q^{b}\right) & \beta \equiv \delta q^{b} r^{c}\left(\bmod q^{b}\right) & \gamma \equiv 1\left(\bmod q^{b}\right) \\
\alpha \equiv 1\left(\bmod r^{c}\right) & \beta \equiv 1\left(\bmod r^{c}\right) & \gamma \equiv \delta q^{b} r^{c}\left(\bmod q^{b}\right) .
\end{array}
$$

We define the $L$ representations

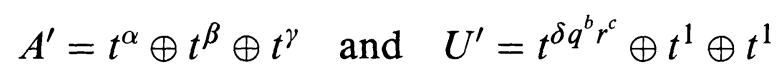

As $\alpha, \beta$, and $\gamma$ are each prime to two of the primes $p, q$, and $r$, no isotropy group of $A^{\prime}-0$ has prime power index in $L$. The isotropy groups of $U^{\prime}-0$ are the cyclic subgroup of $L$ of order $q^{b} r^{c}$ and the trivial group. Consider the action of $G$ on $A^{\prime}$ and $U^{\prime}$ induced by the map $G \rightarrow L$. We denote the representations of $G$ by $A_{2}(K)$ and $U_{2}(K)$. Then

(4) $A_{2}(K)$ satisfies (1), $A_{2}(K)$ and $U_{2}(K)$ satisfy (2), and $K$ is an isotropy group of $U_{2}(K)$. The other isotropy groups of $U_{2}(K)$ are $G$ and $H$. 
Next consider $K \subset G$ such that $|G / K|=p^{d}$, but $G / K$ need not be cyclic, and $d \geq 1$. Write $G / K$ as a product of cyclic groups $C_{1} \times \cdots \times C_{m}$, and let $K_{j}$ be the kernel of the composite map $G \rightarrow$ $G / K \rightarrow C_{j}$. The respective groups $H$ in the diagram of Step 1 are then called $H_{j}$. Observe that

$$
K=\bigcap_{j} K_{j} \quad \text { for } 1 \leq j \leq m .
$$

In Step 1 we constructed for each $K_{j}$ representations $A_{2}\left(K_{j}\right)$ and $U_{2}\left(K_{j}\right)$ of $G$. We set

$$
A_{2}(K)=\bigoplus_{j} A_{2}\left(K_{j}\right) \quad \text { and } \quad U_{2}(K)=\bigoplus_{j} U_{2}\left(K_{j}\right)
$$

Thus for any $K \subset G$ with $|G / K|$ of prime power order we constructed representations $A_{2}(K)$ and $U_{2}(K)$. By construction, the set of isotropy groups of $U_{2}\left(K_{j}\right)$ is $\left\{G, K_{j}, H_{j}\right\}$. Remember that $H_{j} \subseteq K_{j} \subseteq K$. The isotropy groups of $U_{2}(K)$ are the intersections of the isotropy groups of the various $U_{2}\left(K_{j}\right)$ 's. In particular, $K$ is an isotropy group of $U_{2}(K)$. It follows easily from (4) that $A_{2}(K)$ and $U_{2}(K)$ have the properties which we claimed in the lemma.

Corollary 5.5. Let $G$ be a group and $A$ and $B$ real representations of $G$ as in 5.1 (that is, they satisfy Condition 1.1). Let $N$ be an integer. There exists a complex representation $U_{0}$ and a collection $\mathscr{S}_{0}$ of complex representations of $G$ and of cardinality at least $N$ such that

(1) The set of isotropy groups of $U$ is the set of all subgroups of $G$, and $\operatorname{dim} U^{K}=0$ or $\geq 6$ for all $K \subset G$.

(2) For $V \in \mathscr{S}_{0}$ the set of isotropy groups of $V$ consists of $G$ and all subgroups $K \subset G$ such that $G / K$ is not of prime power order. Furthermore, $V^{G}=0$ and $\operatorname{dim} V^{K} \geq 6$ for the other isotropy groups of $V$.

(3) $\operatorname{Res}_{P} V \cong \operatorname{Res}_{P} U$ for all $P \subset G$ of prime power order and $V \in \mathscr{S}_{0}$.

(4) $\operatorname{dim} V^{K}=\operatorname{dim} W^{K}$ for all $K \subset G$ and all $V, W \in \mathscr{S}_{0}$.

(5) If $V, W \in \mathscr{S}_{0}$, then $V-W$ is a non-zero multiple of $A-B$.

The following representation will be useful

$$
\Omega=\mathbb{R}[G]-\sum \psi
$$


where $\mathbb{R}[G]$ is the real regular representation of $G$ and the sum ranges over all irreducible representations $\psi$ of $G$ such that the kernel $G(\psi)$ of $\psi$ is of prime power index in $G$. It is trivial to observe

Proposition 5.7. The representation $\Omega$ is stable and satisfies the gap hypothesis (by a certain margin), and the set of isotropy groups consists of $G$ (with $\Omega^{G}=0$ ) and all subgroups of $G$ not of prime power index.

The remark that stability and gap hypothesis hold by a certain margin means that the inequalities in the definitions of the properties are not sharp, but the slack in about the dimension of $\sum \psi$ from 5.6, and some times larger. The properties of $\Omega$ are easily obtained from those of the regular representation $\mathbb{R}[G]$.

Proof of Corollary 5.5. We set

$$
\begin{aligned}
& V_{j}^{\prime}=j A \oplus(N-j) B \oplus \bigoplus_{K} A_{2}(K) \oplus \Omega, \\
& U_{0}^{\prime}=N A \oplus \bigoplus_{K} U_{2}(K) \oplus \Omega .
\end{aligned}
$$

The sum ranges over all subgroups $K$ of $G$ which are of prime power index in $G$. We complexify these representations, and possibly take multiples to assure that all $\operatorname{dim} U^{K}$ are 0 or $\geq 6$, for all $K \subset G$. The resulting representations are called $V_{j}$ and $U_{0}$, and $\mathscr{S}_{0}=$ $\left\{V_{j} \mid 0 \leq j \leq N\right\}$. It is easy to verify that they have the desired properties.

The properties in the conclusion of Corollary 5.5 and those required in 5.2 differ insofar as we require in 5.2 in addition that $U$ and $V_{j} \in$ $\mathscr{S}$ are stable and satisfy the gap hypothesis. This we will achieve by constructing a representation $U_{1}$ and a representation $D$ such that

$$
U=U_{0} \oplus U_{1} \text { and } \mathscr{S}=\left\{V \oplus D \mid V \in \mathscr{S}_{0}\right\}
$$

have all properties required in 5.2. Here $U_{0}$ and $\mathscr{S}_{0}$ are as in the conclusion of 5.5.

We will have to deal with two situations. The first one will be easy to handle, but we need to prepare the second one. Let $J \subset G$ and $x \in U_{0}$ such that $G_{x}=J$. Let $\psi^{\prime}$ be an irreducible representation of $J$, and let $\psi$ be a representation of $G$ which restricts to $\psi^{\prime}$, so $\operatorname{Res}_{J} \psi=\psi^{\prime}$. Such a $\psi$ always exists. 
LEMMA 5.8. Suppose $G / J$ is of prime power order, and $\psi^{\prime}$ is such that for $\psi$ as above the kernel $G(\psi)$ is of prime power index in $G$. There exist representations $E$ and $F$ of $G$ such that

(1) $\operatorname{Res}_{P} E \cong \operatorname{Res}_{P}(F \oplus \psi)$ for all subgroups $P$ of $G$ of prime power order.

(2) The isotropy groups of $E-0$ and of $F-0$ are not of prime power index in $G$.

Proof. Let $\psi$ be an irreducible representation of $G$ which restricts to $\psi^{\prime}$. Denote the kernel $G(\psi)$ of $\psi$ by $K$. We may suppose that $G / K$ is cyclic of prime power order. Then we get an exact sequence

$$
1 \rightarrow K \rightarrow G \rightarrow G / K=\mathbb{Z}_{p^{a}} \rightarrow 1
$$

where $G / K$ is cyclic and of prime power order. We may then write $\psi$ as a representation $t^{\delta}$ of $\mathbb{Z}_{p^{a}}$, with an induced action of $G$ through the map $G \rightarrow G / K$. The notation $t^{\delta}$ was introduced for a cyclic group in $\S 2$, and by assumption $\delta$ is prime to $p$. We complete above sequence to a diagram as in Step 1 of the proof of Lemma 5.4, using the same notation. The argument of that proof provides us with

$$
A^{\prime}=t^{\alpha} \oplus t^{\beta} \oplus t^{\gamma} \quad \text { and } \quad F^{\prime}=t^{1} \oplus t^{1}
$$

for a cyclic group $L$ whose order is divisible by at least three primes. The representations $A^{\prime}$ and $U^{\prime}=\psi \oplus F^{\prime}$ are as in the construction in that proof. (A small modification of the argument in 5.4 is required if $\psi$ is the trivial representation.) With the induced action of $G$ we call the representations $A^{\prime}$ and $F^{\prime}$ then $E$ and $F$. The properties satisfied in the conclusion of 5.4 imply those required in 5.8.

To prove Theorem 5.3 we use the following ideas. Let $\mathscr{T}(G)$ denote the set of subgroups of $G$, partially ordered by the relation $K \leq H$ if and only if $K \supseteq H$. A subset $\mathscr{T}_{0} \subset \mathscr{T}(G)$ is called closed if $H \leq K$ and $K \in \mathscr{T}_{0}$ implies that $H \in \mathscr{T}_{0}$.

Proof of Theorem 5.3. In Corollary 5.5 we constructed a complex representation $U_{0}$ and a collection of complex representations $\mathscr{S}$ of $G$ such that most of the properties in Theorem 5.3 are satisfied. In addition, only the stability assumption and the gap hypothesis need to be satisfied. This is done inductively with the help of Lemma 5.8 and the representation $\Omega$. 
Consider a closed subset $\mathscr{T}_{0}$ of $\mathscr{T}(G)$ such that the stability assumption holds for all $x \in U_{0}$ for which $G_{x} \in \mathscr{T}_{0}$. Let $K$ be minimal in $\mathscr{T}(G)-\mathscr{T}$, and let $\psi^{\prime}$ be an irreducible representation of $K$ which does not occur often enough in $\operatorname{Res}_{K} U_{0}$. Suppose $\psi$ is an irreducible representation of $G$ which restricts to $\psi^{\prime}$. If $G(\psi)$ is of prime power index in $G$, then we add sufficiently many copies of $E$ to each of the elements in $\mathscr{S}_{0}$ and an equal number of copies of $F \oplus \psi$ to $U_{0}$ (see Lemma 5.8). If $G(\psi)$ is not of prime power index in $G$, we add sufficiently many copies of $\Omega$ to the elements in $\mathscr{S}_{0}$ and to $U_{0}$. This will assure that $\psi^{\prime}$ will occur often enough in $\operatorname{Res}_{K} U_{0}$ and in this process the inductive assumption continues to hold. (To avoid excessive notation we use the same notation before and after these additions.) In this process we can assume that $U_{0}$ becomes stable.

To assure that each $V \in \mathscr{S}_{0}$ becomes stable we only need to add copies of $\Omega$ to all $V \in \mathscr{S}_{0}$ and to $U_{0}$. In most cases stability implies the gap hypothesis, but the same procedure as above can also be used to achieve this.

The inductive application of this procedure adds a representation $\mathscr{E}$ to all $V \in \mathscr{S}_{0}$ ( $\mathscr{E}$ is the sum of all those $E$ and $\Omega$ required above) and $\mathscr{F}$ to $U_{0}$ ( $\mathscr{F}$ is the sum of all the $F \oplus \psi$ and $\Omega$ required above). It is clear that

$$
U=U_{0} \oplus \mathscr{F} \quad \text { and } \quad \mathscr{S}=\left\{V \oplus \mathscr{E} \mid V \in \mathscr{S}_{0}\right\}
$$

has all of the desired properties.

\section{Proof of Theorem $B$.}

Sketch of Proof of Theorem B. The starting point is a representation $U$ and a sufficiently large collection $\mathscr{S}$ of representations of $G$ which satisfy Condition 5.2. Non-isomorphic representations $A$ and $B$ of $G$ which satisfy Condition 1.1 (see the introduction) can be constructed, and from such $A$ and $B$ we can construct $U$ and $\mathscr{S}$. We showed this in the last section.

In Theorem 6.3 we use $\mathscr{S}$ and $U$ to produce manifolds $X(V, W)$ for $V$ and $W$ in $\mathscr{S}$ which have exactly two fixed points at which the tangent representations are $V$ and $-W$ (the - sign indicates a reversed orientation). These manifolds satisfy a few technical assumptions which are derived from those of the representations in $\mathscr{S}$.

There is a sequence of obstructions $\sigma_{P}$ for finding an equivariant cobordism (relative to the fixed point set) between $X(V, W)$ and a homotopy sphere. These will lie in finite obstruction groups, and with 
respect to connected sum these obstructions are additive. A reversal of orientation changes the sign of $\sigma_{P}$. Starting out with a set $\mathscr{S}$ of sufficiently large cardinality, these thoughts together with a pigeon hole principle will provide manifolds $X(V, W)$ for which all $\sigma_{P}$ vanish. We may then use $X(V, W)$ as a homotopy sphere with exactly two fixed points and Smith equivalent tangent representations $V$ and $W$.

Our first step is to introduce the surfaces from $\S 3$ into our context. As before, let $G$ be a finite abelian group and $\psi$ an irreducible representation of $G$. We defined the kernel $G(\psi)$ of $\psi$ and $L(\psi)=G / G(\psi)$, which is always a cyclic group (see [S, Chapter 3 Exercise $3.2(\mathrm{c})])$. We suppose that $|L(\psi)|=m(\psi)$ is not of prime power order. We choose a decomposition $m(\psi)=r(\psi) s(\psi)$ where $r(\psi)$ and $s(\psi)$ depend only on $m(\psi)$ and not on $\psi$. We also choose $a(\psi)$ and $b(\psi)$ such that $a(\psi) r(\psi)+b(\psi) s(\psi)+1 \equiv 0(\bmod m(\psi))$, and we set

$$
A_{+}(\psi)=b(\psi) \cdot\left[\mathbb{Z}_{m(\psi)} / \mathbb{Z}_{r(\psi)}\right] \sqcup a(\psi) \cdot\left[\mathbb{Z}_{m(\psi)} / \mathbb{Z}_{s(\psi)}\right] \sqcup\left[\mathbb{Z}_{m(\psi)} / \mathbb{Z}_{m(\psi)}\right] .
$$

Our choice is slightly less delicate than the one in 3.2 .

As in $\S 3$ (see the paragraph before Lemma 3.3) we get a surface $X^{\prime}(\psi)$ with smooth $L(\psi)$ action. The projection $G \rightarrow L(\psi)$ induces a $G$ action on this surface, and then we denote it by $X(\psi)$ as in Lemma 3.3.

Let $V$ be a representation of $G$ such that $V^{K}=0$ whenever $G / K$ is of prime power index in $G$. Each irreducible summand $\psi$ of $V$ is such that $|L(\psi)|$ is divisible by at least two primes, and $X(\psi)$ is defined. As in 3.4 we set for $V=\sum a_{\psi}(V) \psi$

$$
X(V)=\prod X(\psi)^{a_{\psi}(V)} .
$$

The properties of $X(V)$ are described in our next lemma (compare Corollary 3.5 and Theorem 3.7).

LEMMA 6.2. Let $G$ be an abelian group and $\mathscr{S}$ a collection of representations of $G$ as in 5.2. There is a collection of closed $G$ oriented manifolds $\{X(V) \mid V \in \mathscr{S}\}$ such that

(1) $X(V)^{G}=x$ consists of exactly one point and $T_{x} X(V)=V$ as oriented $G$ representation.

(2) There is a representation $C$ of $G$ such that $T X(U) \oplus \underline{C}$ and $\underline{U} \oplus \underline{C}$ are isomorphic $G$ vector bundles.

(3) $\chi\left(X(D)^{K}\right)=\chi\left(X(E)^{K}\right)$ for all $K \subset G$ and $D, E \in \mathscr{S}$. 
(4) For all $K \subset G$ not of prime power index $\operatorname{Res}_{K} X(V)$ is a $K$ equivariant boundary. There is a stable $K$ equivariant trivialization of the tangent bundle of this zero cobordism which extends the stable trivialization of $T X(V)$ in (2), after restricting this action to a $K$ action.

(5) $\operatorname{Sign}\left(G, X(V)^{P}\right)=0$ for all $V \in \mathscr{S}$ and $P$ of prime power order.

Proof. Properties (1), (2), and (4) have been discussed in $\S 3$ and its Addendum. We discuss (3). Let $\psi$ be irreducible. According to Proposition 3.1 the Euler characteristic $\chi\left(X(\psi)^{K}\right)$ depends only on $L(\psi)$ and the choice of $A_{ \pm}(\psi)$, hence only on $L(\psi)$. Thus $\chi\left(X(\psi)^{K}\right)$ depends only on $\left\{\left(\operatorname{dim} V^{K}, K\right) \mid K \subset G\right\}$. By assumption $\operatorname{dim} V^{K}=$ $\operatorname{dim} W^{K}$ for all $K \subset G$, and (3) follows.

To see (5) we observe that $G$ acts on $X(V)^{P}$, and any cyclic subgroup $C$ of $G$ generates together with $P$ a subgroup $K$ which is not of prime power index in $G$. But $\operatorname{Res}_{K}\left(X(V)^{P}\right)$ bounds $K$ equivariantly according to (4). As a bordism invariant the signature vanishes.

For the idea of a Smith framed manifold we refer the reader once more to [PR1] or [DP2]. This concept enters in the technical steps of equivariant surgery which do not concern us here. Property (2) in our next theorem is obtained from the assumption that $\operatorname{Res}_{P} U \cong \operatorname{Res}_{P} V$ for all $P \subset G$ of prime power order. It is important that these are complex representations and that this isomorphism is one of complex representations.

In our next proof we will make reference to the Burnside ring $\Omega(G)$ of $G$. Its elements are represented by finite $G C W$ complexes. Two finite $G C W$ complexes $X$ and $Y$ are equivalent in $\Omega(G)$ if and only if $\chi\left(X^{K}\right)=\chi\left(Y^{K}\right)$ for all $K \subset G$. The class of $X$ in $\Omega(G)$ is denoted by $[X]$. Addition is given by disjoint union of representative complexes, and multiplication by their cartesian product. A single point with trivial action represents 1 in this ring.

THEOREM 6.3. Let $U$ and $\mathscr{S}$ be as in 5.2. There are smooth $G$ oriented manifolds $X=X(V, W)$ for $V$ and $W \in \mathscr{S}$ such that

(1) $X$ is stable and satisfies the gap hypothesis.

(2) $X$ is $U$ Smith framed.

(3) $X^{G}=\{x, y\}$ consists of exactly two points and $T_{X} X=V$ and $T_{y} X=-W$.

(4) $\chi\left(X^{K}\right)=2$ and $\operatorname{dim} V^{K} \equiv 0(\bmod 4)$ for all $K \subset G$. 
(5) $\operatorname{Res}_{K} X$ is $U$ Smith framed cobordant to zero whenever $G / K$ is not of prime power order and $\operatorname{Sign}\left(G, X^{P}\right)=0$ whenever $P$ is a subgroup of $G$ of prime power order.

Proof. By assumption $\chi\left(X(V)^{K}\right)=\chi\left(X(W)^{K}\right)$ for all $K \subset G$, and in the Burnside ring $[X(V)]=[X(W)]$. Thus $X(V) \sqcup-X(W)$ represents a class in the Burnside ring which is divisible by 2 . We recall from [DP2, page 303] how to construct an equivariantly stably parallizable boundary $Z$ from the representation $U$ such that $X(V, W)=$ $X(V) \sqcup-X(W) \sqcup Z$ satisfies (4). Let $Y$ be a closed oriented surface with trivial $G$ action and $\chi(Y)=-2$. Write $U=U^{\prime} \oplus \mathbb{R}^{2}$. Set

$$
Z^{+}=S(U \oplus \mathbb{R}) \text { and } Z^{-}=Y \times S\left(U^{\prime} \oplus \mathbb{R}\right) .
$$

We equip the manifolds with a $U$ Smith framing. In the Burnside ring

$$
\left[G \times{ }_{H} Z^{+}\right]=2[G / H] \text { and }\left[G \times_{H} Z^{-}\right]=-4[G / H] \text {. }
$$

It is now obvious that $[X(V)]+[X(W)]-2$ is a linear combination of the $G \times_{H} Z^{ \pm}$for $H \neq G$.

The other properties follow easily from this definition and those properties listed in 6.2.

Proof of Theorem B (Special Case). If we assume in Theorem B that $G$ is of odd order, then we can complete the proof quickly. We only need a set $\mathscr{S}$ of cardinality 2 and a representation $U$ as in 5.2, and they exist by Theorem 5.3. The manifolds $X=X(V, W)$ satisfy the assumption in the induction theorem for equivariant surgery (see [DP3, Theorem 2.8] or [PR1]). The assumption that $|\mathscr{S}| \geq 2$ assures that we have $X(V, W)$ with $V \neq W$. In the references one deals with normal maps which may be obtained mapping $X(V, W)$ to the sphere $S(V \oplus \mathbb{R})$ via the Thom-Pontrjagin collapse. That is, we collapse the complement of a neighbourhood of the fixed point $x$ at which the tangent representation is $V$. The induction theorem implies that $X$ is equivariantly cobordant to a homotopy sphere relative to the fixed point set. This shows that $V$ and $W$ are Smith equivalent.

The special case is only a partial technical improvement of [PR1] based on the additional information about the manifolds $X(V, W)$ in Theorem 6.3. We proceed with the preparation of the general proof. 
Let $\Lambda$ be $\mathbb{Z}$ inverted at a finite number of primes. Consider the kernel of the equivariant signature map

$$
I(\Lambda[H])=\operatorname{ker}\left(L_{0}^{h}(\Lambda[H], 1) \stackrel{\text { Sign }}{\longrightarrow} R(H)\right)
$$

which takes values in the representation ring of $H$. In our situation $H$ is a finite abelian group. We thank A. Bak for providing us with the details for the following result. They are spelled out in the next section.

\section{THEOREM 6.5. $I(\Lambda[H])$ is finite.}

Let $\Lambda=\mathbb{Z}\left[1 / q_{1}, \ldots, 1 / q_{k}\right]$ be the ring obtained by inverting the primes $q_{1}, \ldots, q_{k}$, and let $p$ be a prime different from $q_{j}$. Let

$$
J(\Lambda, p, H)=\operatorname{ker}\left(\widetilde{K}_{0}(\Lambda[H]) \rightarrow \widetilde{K}_{0}\left(\mathbb{Z}_{(p)}[H]\right)\right) .
$$

Proposition 6.6. There exist primes $q_{k+1}, \ldots, q_{k+r}$ different from $p$ such that for $\Lambda^{\prime}=\mathbb{Z}\left[1 / q_{1}, \ldots, 1 / q_{k+r}\right]$

$$
J(\Lambda, p, H)=\operatorname{ker}\left(\widetilde{K}_{0}(\Lambda[H]) \rightarrow \widetilde{K}_{0}\left(\Lambda^{\prime}[H]\right)\right) .
$$

So we only need to invert finitely many additional primes (different from $p$ ) such that each element in $J(\Lambda, p, H)$ becomes stably free. This follows easily as $\widetilde{K}_{0}(\Lambda[H]$ ) is finite. (For a proof see also Lemma 7.1.)

The proof of Theorem B will be inductive, and we describe the situation which we will encounter in all but the last step.

Consider $f: X \rightarrow Y$, where $X$ and $Y$ are smooth $G$ oriented manifolds, $G$ is a finite abelian group, and $f$ is equivariant. Suppose these manifolds are stable and satisfy the gap hypothesis, $X$ is Smith framed, and every subgroup $P \subset G$ of prime power order occurs as isotropy group of $X$. We suppose that $X^{P}$ and $Y^{P}$ are connected and $\operatorname{dim} X^{P}=\operatorname{dim} Y^{P} \equiv 0(\bmod 4)$ and $\geq 6$. We assume that $Y^{P}$ is simply connected, and in our application $Y$ will actually be the unit sphere in a representation. Furthermore, suppose that $f^{P}$ is of degree 1. We suppose that $\operatorname{Sign}(G, X)=\operatorname{Sign}(Y, G)=0$.

The data $(X, f, b)$ describe a normal map. Here $f: X \rightarrow Y$ is as above and $b$ is a Smith framing. These data need to satisfy a few conditions to be called a normal map, and all of them follow from the assumptions listed in the last paragraph and Condition 6.7 (1). Associated to these normal maps there is a natural concept of a Smith framed 
normal cobordism between Smith framed normal maps. The cobordism is supposed to have the same type of data as the normal maps. In particular, it induces an equivariant cobordism between the domains of the normal maps. As before we hope the reader knows these concepts or will read them in the literature on equivariant surgery. The most appropriate references in our setting are [DP2] and [PR2] because there normal maps with Smith bundle data are treated. But, the reader only needs to know that normal maps are objects equivariant surgery can be applied to, and as we are only going to quote the necessary results from equivariant surgery without proof no more detailed knowledge is required to follow the proof.

Given a closed subset $\mathscr{T}_{0}$ of the set $\mathscr{T}(G)$ of all subgroups of $G$. The definition is given after Lemma 5.7. Let $(X, f, b)$ be as described in the last two paragraphs. We will encounter the following

\section{Inductive Assumption 6.7. (1) $\chi\left(X^{K}\right)=\chi\left(Y^{K}\right)$ for all $K \subset G$.}

(2) Let $P \in \mathscr{T}_{0}$ be a subgroup of $G$ of $p$ power order, $p$ a prime. We are given a finite collection of primes $q_{1}, \ldots, q_{k}$ different from $p$ and $f^{P}$ induces a homology isomorphism with coefficients in $\Lambda=$ $\mathbb{Z}\left[1 / q_{1}, \ldots, 1 / q_{k}\right]$. In particular, $f^{P}$ is a homology isomorphism with $\mathbb{Z}_{p}$ coefficients. This collection of primes depends on the groups $P^{\prime} \leq P$ of $p$ power order.

Let $P$ be a minimal element in $\mathscr{T}(G)-\mathscr{T}_{0}$ of $p$ power order. We shall discuss the obstruction for finding a Smith framed normal cobordism between $(X, f, b)$ and another Smith framed normal map which satisfies the Inductive Assumption 6.7 for $\mathscr{T}_{0} \cup\{P\}$.

TheOREM 6.8. Suppose $\mathscr{T}_{0}$ and $(X, f, b)$ as in 6.7 and $P \neq 1$ is a minimal element in $\mathscr{T}(G)-\mathscr{T}_{0}$ of $p$ power order, $p$ a prime. There exist primes $r_{1}, \ldots, r_{s}$ different from $p$ and an obstruction $\sigma_{P}(f, b) \in I(\Lambda[G / P])$ with $\Lambda=\mathbb{Z}\left[1 / r_{1}, \ldots, 1 / r_{s}\right]$ which has the following property. If $\sigma_{P}(f, b)=0$ then $(X, f, b)$ is Smith framed normally cobordant to another Smith framed normal map $\left(X^{\prime}, f^{\prime}, b^{\prime}\right)$ such that the inductive assumption holds for $\mathscr{T}_{0} \cup\{P\}$. The cobordism is relative to all $K$ fixed point sets such that $K$ is not a subgroup of $P$.

Before we begin with the proof we fix a bit more notation. We let $M$ denote the mapping cylinder. In the fixed point set $X^{P}$ we consider the singular set $X^{P, s}=\left\{x \in X^{P} \mid G_{x} \neq P\right\}$. Similarly, $f^{P, s}$ 
is the induced map from $X^{P, s}$ to $Y^{P, s}$. We also set

$$
\begin{aligned}
K_{*}\left(X^{P}, \Lambda\right) & =H_{*+1}\left(M_{f^{P}}, X^{P} ; \Lambda\right) \\
& =\operatorname{ker}\left(H_{*}\left(X^{P}, \Lambda\right) \rightarrow H_{*}\left(Y^{P}, \Lambda\right)\right) .
\end{aligned}
$$

The second equality follows from the assumption that $f^{P}$ has degree 1 . We note

$$
H_{*}\left(M_{f^{P, s}}, X^{P, s} ; \Lambda\right)=0
$$

whenever $P$ is not a Sylow subgroup, the inductive assumption holds for $\mathscr{T}$, and $P$ is minimal in $\mathscr{T}(G)-\mathscr{T}_{0}$. Here $\Lambda$ is $\mathbb{Z}\left[1 / \tilde{q}_{1}, \ldots, 1 / \tilde{q}_{k}\right]$ such that the set $\left\{\tilde{q}_{1}, \ldots, \tilde{q}_{k}\right\}$ contains all those primes $q_{j}$ which occur in 6.7 (2) for $P^{\prime} \in \mathscr{T}_{0}$ such that $P^{\prime}<P$. This follows from the universal coefficient theorem and a Mayer-Vietoris argument. Note in particular that the $p$ Sylow subgroup $G_{p}$ contains all groups $P^{\prime}$ of $p$ power order which contain $P$.

Let $P$ be the chosen element in $\mathscr{T}(G)-\mathscr{T}_{0}$ and let $P$ be of $p$ power order. The set $\omega(P)=\left\{r_{1}, \ldots, r_{s}\right\}$ will be the union of two sets, $\omega(P)=\omega_{1} \cup \omega_{2}$. We define $\omega_{1}$. If $P$ is the $p$ Sylow subgroup we let $\omega_{1}$ be the set of prime divisors of $|G / P|$. If $P$ is not the $p$ Sylow subgroup we consider the sets $\omega\left(P^{\prime}\right)=\left\{q_{1}, \ldots, q_{k}\right\}$ which have been defined before for $P^{\prime}<P$ of $p$ power order (see 6.7 (2)). Then we set $\omega_{1}=\bigcup_{P^{\prime}<P} \omega\left(P^{\prime}\right)$.

The set $\omega_{2}$ consists of those primes $q_{k+1}, \ldots, q_{k+r}$ in Proposition 6.6 which need to be inverted such that the elements in $J(\Lambda, p, G / P)$ become stably free if these primes are inverted as well. Now we set $\omega(P)=\omega_{1} \cup \omega_{2}$. We set $\Lambda_{0}=\mathbb{Z}\left[1 / r_{1}, \ldots, 1 / r_{k}\right]$ with $r_{1}, \ldots, r_{k} \in$ $\omega_{1}$ and $\Lambda=\mathbb{Z}\left[1 / r_{1}, \ldots, 1 / r_{s}\right]$ with $r_{1}, \ldots, r_{s} \in \omega$. Because $\omega\left(P^{\prime}\right)$ is finite, $\omega(P)$ is finite as well.

Proof of Theorem 6.8, Part 1. In the first part of the proof we show that after surgery below the middle dimension $(2 \mathrm{~m})$ we may suppose that $K_{2 m}\left(X^{P}, \Lambda\right)$ is $\Lambda[G / P]$ stably free.

Let $\mathscr{W}=(X, f: X \rightarrow Y, b)$ be the normal map and $P$ the chosen minimal element in $\mathscr{T}(G)-\mathscr{T}_{0}$. Suppose that $X^{P}$ is $4 m$-dimensional (see 6.3 (4)). There is a normal map $\mathscr{W}_{1}=\left(X_{1}, f_{1}: X_{1} \rightarrow Y_{1}, b_{1}\right)$ which is $G$ normally cobordant to $\mathscr{W}$ (relative to all $H$ fixed point set such that $H$ is not a subgroup of $P$ ) such that $f_{1}^{P}$ is connected up to the middle dimension. So $f_{1}^{P}$ is $2 m$-connected. Such a cobordism and $\mathscr{W}_{1}$ can be constructed by equivariant surgery as in [DP1], and for Smith bundle data one uses in addition the reference to [PR1]. In 
particular, $K_{2 m}\left(X_{1}^{P}, \mathbb{Z}\right)$ is the only non vanishing kernel in homology and this module is torsion free. We distinguish two cases.

Case 1. Let $P$ be the $p$ Sylow subgroup. We defined $\Lambda_{0}$ above. As we inverted all divisors of $|G / P|$ it follows that $K_{2 m}\left(X_{1}^{P}, \Lambda_{0}\right)$ is a semi simple $\Lambda_{0}[G / P]$ module, and thus it is projective over $\Lambda_{0}[G / P]$.

Case 2. Suppose $P$ is not the $p$ Sylow subgroup. By the inductive assumption and the definition of $\Lambda_{0}$ the chain complex $C_{*}\left(M_{f_{1}^{P^{\prime}}}, X_{1}^{P^{\prime}}\right) \otimes \Lambda_{0}$ is acyclic for all $P^{\prime}<P$ of $p$ power order. Thus $C_{*}\left(M_{f_{1}^{P, s}}, X_{1}^{P, s}\right) \otimes \Lambda_{0}$ is acyclic and

$$
\begin{aligned}
K_{*-1}\left(X_{1}^{P}, \Lambda_{0}\right) & \cong H_{*}\left(M_{f_{1}^{P}}, X_{1}^{P} ; \Lambda_{0}\right) \\
& \cong H_{*}\left(M_{f_{1}^{P}}, M_{f_{1}^{P, s}} \cup X_{1}^{P} ; \Lambda_{0}\right) \\
& \cong H\left(C_{*}\left(M_{f_{1}^{P}}, M_{f_{1}^{P, s}} \cup X_{1}^{P}\right) \otimes \Lambda_{0}\right)
\end{aligned}
$$

which is the homology of a $\mathbb{Z}[G / P]$ free chain complex. This chain complex has only one non vanishing homology group (in dimension $2 m+1)$ and is then projective over $\Lambda_{0}[G / P]$.

We return to the general case. It follows from Oliver's work [O] that the Euler characteristic assumption made in 6.7 (1) implies that $K_{2 m}\left(X_{1}^{P}, \mathbb{Z}_{(p)}\right)$ is stably $\mathbb{Z}_{(p)}[G / P]$ free. It follows from the definition of $\Lambda$ and from Proposition 6.6 that $K_{2 m}\left(X_{1}^{P}, \Lambda\right)$ is stably $\Lambda[G / P]$ free. This is what we wanted to show in the first part of this proof.

Part 2. Let $\lambda$ and $\mu$ be the intersection and self intersection form defined as by Wall [W]. These forms are tensored with $\Lambda$ as indicated from context. By definition $\left(K_{2 m}\left(X_{1}^{P}, \Lambda\right), \lambda, \mu\right)$ is a $\Lambda[G / H]$ hermitian form which represents a class $\sigma_{P}\left(f_{1}, b_{1}\right) \in L_{0}^{h}(\Lambda[G / H], 1)$. By assumption $\operatorname{Sign}(G, X)-\operatorname{Sign}(G, Y)=\operatorname{Sign}\left(G,\left(K_{2 m}\left(X_{1}^{P}\right), \lambda\right)\right)=0$. Thus $\sigma_{P}\left(f_{1}, b_{1}\right)$ is an element in $I(\Lambda[G / P]$ ) (see 6.4). Actually, $\sigma_{P}\left(f_{1}, b_{1}\right)$ depends only on $(f, b)$ which is expressed by the notation $\sigma_{P}(f, b)$.

Part 3. Suppose $\sigma_{P}(f, b)=0$. It follows from standard procedures in equivariant surgery theory that the middle dimensional surgery kernel $K_{2 m}\left(X_{1}^{P}, \Lambda\right)$ can be killed. The function $f_{2}^{P}$ of the resulting normal map $\mathscr{W}_{2}=\left(X_{2}, f_{2}: X_{2} \rightarrow Y_{2}, b_{2}\right)$ (which is equivariantly cobordant to $\mathscr{W}_{1}$ ) induces a homology isomorphism. In the adjustment which we made to the normal map we disturbed $\chi\left(X^{P^{\prime \prime}}\right)$ for $P^{\prime \prime}>P$. They can be adjusted by 0 and 1 dimensional surgeries on 
the $P^{\prime \prime}$ fixed point set. This is easy based on the well known effects of surgery on Euler characteristics.

These three parts complete the proof of Theorem 6.8.

In our proof of Theorem B we need an integer $N$. Set $N=$ $\prod_{P}(N(P)+1)$ where $P$ is of prime power order or $P=1$. Here $N(P)$ is the order of $I(\Lambda[G / P])$ in 6.4 and $\Lambda$ is as in 6.7 (2). For $P=1$ we set

$$
I(\mathbb{Z}[G])=\operatorname{ker}\left(L_{0}^{B_{0}(G)}(\mathbb{Z}[G], 1) \stackrel{\text { Sign }}{\longrightarrow} R(G)\right) .
$$

The group $B_{0}(G)$ occurs in the work of Oliver and Petrie [O] and [OP]. For its use in this context see the proof of Theorem B in [DP2] or $\S 6$ of [DP1]. It is a finite subgroup of $\widetilde{K}_{0}(\mathbb{Z}[G])$, so $I(\mathbb{Z}[G])$ is finite. Then $N(1)$ is the order of $I(\mathbb{Z}[G])$.

We are now ready to give the

Proof of Theorem B. We start out with a representation $U$ and a collection $\mathscr{S}$ of representations of $G$ as in 5.3 of cardinality at least $N$ (as above). From these we construct the manifolds $X=X(V, W)$ as in Theorem 6.3. To each such manifold we assign the Smith framed normal map $(X, f: X \rightarrow Y, b)$. Here $Y=S(V \oplus \mathbb{R}), f$ is the ThomPontrjagin collapse from $X$ to $Y$, and $b$ is the Smith framing of $X$.

We now do an induction over the set $\mathscr{P}(G)$ of subgroups of $G$ of prime power order, including 1 . Let $\mathscr{T}$ be a closed subset of $\mathscr{T}(G)$ as in 6.7, and say that $X(V, W)$ is $\mathscr{T}_{0}$ adjusted if $X(V, W)$ satisfies the assumptions in 6.7 for this set $\mathscr{T}_{0}$. For each $\mathscr{T}_{0}$ adjusted manifold $X(V, W)$ and minimal $P \in \mathscr{T}(G)-\mathscr{T}_{0}$ we have the obstruction $\sigma_{P}(f, b)$ in $I(\Lambda(G / P))$ (see 6.8). We denote it by $\sigma_{P}(X(V, W))$. The obstruction has the same properties as those listed for $\sigma$ in 4.6.

Furthermore, let a set $\mathscr{S}^{\prime} \subset \mathscr{S}$ of cardinality $M$ be given such that $X(V, W)$ is $\mathscr{T}_{0}$ adjusted. We can find a representation $V$ and representations $W_{j}, 1 \leq j \leq M_{0}=M / N(P)-1$ such that $\sigma_{P}\left(X\left(V, W_{j}\right)\right)=$ $\sigma_{P}\left(X\left(V, W_{i}\right)\right)$ for $1 \leq i, j \leq M_{0}$. As in the proof of Theorem A in $\S 4$ we conclude (based on 4.6) that $\sigma\left(X\left(W_{i}, W_{j}\right)\right)=0$. By 6.8 we may assume that $X\left(W_{i}, W_{j}\right)$ is $\mathscr{T}_{0} \cup\{P\}$ adjusted. In this way we may proceed until we find representations $W_{j}, 1 \leq j \leq N(1)+1$ such that $X\left(W_{i}, W_{j}\right)$ is $\mathscr{T}(G)-\{1\}$ adjusted.

Again we get a pair of non isomorphic representations $W_{1}$ and $W_{2}$ such that

$$
0=\sigma\left(X\left(W_{1}, W_{2}\right)\right) \in L_{0}^{B_{0}(G)}(\mathbb{Z}[G], 1) .
$$


It is explained in [DP2, Theorem E (ii), page 301] how to apply equivariant surgery and find an equivariant cobordism between $X\left(W_{1}, W_{2}\right)$ and a homotopy sphere $\Sigma$, relative to the fixed point set. Then $W_{1} \neq W_{2}$ and these representations are Smith equivalent.

Because $W_{1}$ and $W_{2}$ are in $\mathscr{S}$ we may suppose the $W_{1}-W_{2}$ is a multiple of $A-B$, as in the formulation of the theorem.

7. Some algebraic computations (by A. Bak). We thank A. Bak for the computations in this section. To prove Theorem 6.5 , a lemma is required. Let $\widetilde{K}_{0}(\mathbb{Z}[H])=K_{0}(\mathbb{Z}[H]) /[\mathbb{Z}[H]]$.

LEMMA 7.1. $\widetilde{K}_{0}(\mathbb{Z}[H])$ is finite.

Proof. By a theorem of Swan [Sw], if $P$ is a finitely generated, projective $\mathbb{Z}[H]$-module then $P \otimes_{\mathbb{Z}[H]} \mathbb{Q}[H]$ is a free $\mathbb{Q}[H]$-module. If $\operatorname{rank}(P)$ denotes the rank of this $\mathbb{Q}[H]$-module then it is an easy exercise to check that the sequence

$$
\widetilde{K}_{0}(\mathbb{Z}[H]) \rightarrow K_{0}(\mathbb{Z}[H]) \rightarrow K_{0}(\mathbb{Q}[H])
$$

is exact. The first homomorphism maps $[P]$ to $[P]-\operatorname{rank}(P) \cdot[\mathbb{Z}[H]]$.

Let $\mathscr{O}$ denote the unique maximal $\mathbb{Z}$-order on $\mathbb{Q}[H]$. If

$$
K_{1}(\mathbb{Q}[H]) \rightarrow K_{0}(\mathbb{Z}[H], \mathbb{Q}[H]) \rightarrow K_{0}(\mathbb{Z}[H]) \rightarrow K_{0}(\mathbb{Q}[H])
$$

and

$$
K_{1}(\mathbb{Q}[H]) \rightarrow K_{0}(\mathscr{O}, \mathbb{Q}[H]) \rightarrow K_{0}(\mathscr{O}) \rightarrow K_{0}(\mathbb{Q}[H])
$$

denote respectively the exact $K$-theory localization sequences associated to the homomorphisms $\mathbb{Z}[H] \rightarrow \mathbb{Q}[H]$ and $\mathscr{O} \rightarrow \mathbb{Q}[H]$ then by [BS, (3.1) and (3.2)]

$$
K_{0}(\mathbb{Z}[H], \mathbb{Q}[H]) \cong \coprod_{p} \operatorname{coker}\left(K_{1}\left(\widehat{\mathbb{Z}}_{p}[H]\right) \rightarrow K_{1}\left(\widehat{\mathbb{Q}}_{p}[H]\right)\right)
$$

and

$$
K_{0}(\mathscr{O}, \mathbb{Q}[H]) \cong \coprod_{p} \operatorname{coker}\left(K_{1}\left(\widehat{\mathscr{O}}_{p}\right) \rightarrow K_{1}\left(\widehat{\mathbb{Q}}_{p}[H]\right)\right)
$$

where $\widehat{\mathbb{Z}}_{p}$ and $\widehat{\mathbb{Q}}_{p}$ denote respectively the completions of $\mathbb{Z}$ and $\mathbb{Q}$ at the prime $p$ and $\widehat{\mathscr{O}}_{p}=\mathscr{O} \otimes_{\mathbb{Z}} \widehat{\mathbb{Z}}_{p}$. Since

$$
\widetilde{K}_{0}(\mathbb{Z}[H]) \cong \operatorname{coker}\left(K_{1}(\mathbb{Q}[H]) \rightarrow K_{0}(\mathbb{Z}[H], \mathbb{Q}[H])\right)
$$


and since $\operatorname{coker}\left(K_{1}(\mathbb{Q}[H]) \rightarrow K_{0}(\mathscr{O}, \mathbb{Q}[H])\right)$ splits as a (finite) product $\prod_{i} \mathrm{Cl}\left(F_{i}\right)$ of (finite) ideal class groups $\mathrm{Cl}\left(F_{i}\right)$ (where $\mathbb{Q}[A]=$ $\prod_{i} F_{i}, F_{i}$ cyclotomic number fields), it suffices to show that

$$
\coprod_{p} \operatorname{coker}\left(K_{1}\left(\widehat{\mathbb{Z}}_{p}[H]\right) \rightarrow K_{1}\left(\widehat{\mathscr{O}}_{p}\right)\right)
$$

is finite.

Let $n$ denote the order of $H$. If $p \nmid n$ then $\widehat{\mathbb{Z}}_{p}[H]=\widehat{\mathscr{O}}_{p}$ (cf. [Bs, $\mathrm{XI}(1.2)])$, and so $\operatorname{coker}\left(K_{1}\left(\widehat{\mathbb{Z}}_{p}[H]\right) \rightarrow K_{1}\left(\widehat{\mathscr{O}}_{p}\right)\right)=0$. If $p \mid n$ then for some natural number $r, p^{r} \widehat{\mathscr{O}}_{p} \subset \widehat{\mathbb{Z}}_{p}[H]$. Let $K_{1}\left(\widehat{\mathscr{O}}_{p}, p^{r} \widehat{\mathscr{O}}_{p}\right)$ denote the relative $K_{1}$-group [Bs, V $\S 2$, IX $\S 1$ ] defined by the ideal $p^{r} \widehat{\mathscr{O}}_{p}$. The canonical homomorphism

$$
\operatorname{coker}\left(K_{2}\left(\widehat{\mathscr{O}}_{p}, p^{r} \widehat{\mathscr{O}}_{p}\right) \stackrel{f}{\longrightarrow} K_{1}\left(\widehat{\mathscr{O}}_{p}\right)\right) \rightarrow \operatorname{coker}\left(K_{1}\left(\widehat{\mathbb{Z}}_{p}[H]\right) \stackrel{g}{\longrightarrow} K_{1}\left(\widehat{\mathscr{O}}_{p}\right)\right)
$$

is surjective. But by the exact sequence [Bs, IX (1.2)]

$$
K_{1}\left(\widehat{\mathscr{O}}_{p}, p^{r} \widehat{\mathscr{O}}_{p}\right) \stackrel{f}{\longrightarrow} K_{1}\left(\widehat{\mathscr{O}}_{p}\right) \rightarrow K_{1}\left(\widehat{\mathscr{O}}_{p} / p^{r} \widehat{\mathscr{O}}_{p}\right)
$$

and the fact that the determinant map $K_{1}\left(\widehat{\mathscr{O}}_{p} / p^{r} \widehat{\mathscr{O}}_{p}\right) \rightarrow \operatorname{units}\left(\widehat{\mathscr{O}}_{p} / p^{r} \widehat{\mathscr{O}}_{p}\right)$ is an isomorphism [Bs, $\mathrm{V}(9.2)]$, one obtains that

$$
\operatorname{coker}(f) \longmapsto \operatorname{units}\left(\widehat{\mathscr{O}}_{p} / p^{r} \widehat{\mathscr{O}}_{p}\right) \text {. }
$$

Since $\widehat{\mathscr{O}}_{p} / p^{r} \widehat{\mathscr{O}}_{p} \cong \mathscr{O} / p^{r} \mathscr{O}$ is a finite ring, $\operatorname{coker}(f)$ is finite. Thus, $\operatorname{coker}(g)$ is finite and the proof is complete.

Proof of Theorem 6.5. By a theorem of Bak [Ba1, Theorem 2] and the remark following [Ba1, Theorem 2], there is an exact sequence

$$
H^{2}\left(\widetilde{K}_{0}(\mathbb{Z}[H])\right) \rightarrow L_{0}^{h}(\mathbb{Z}[H], 1) \stackrel{\text { Sign }}{\longrightarrow} \mathbb{Z}^{r} .
$$

Since the diagram

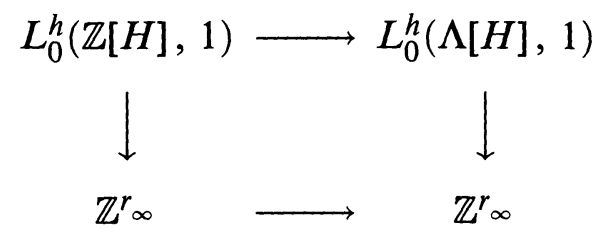

commutes and $H^{2}\left(\widetilde{K}_{0}(\mathbb{Z}[H])\right)$ is finite by the lemma above, it suffices to show that the $\operatorname{coker}\left(L_{0}^{h}(\mathbb{Z}[H], 1) \rightarrow L_{0}^{h}(\Lambda[H], 1)\right)$ is finite.

If $A$ is a ring with involution and $\lambda \in \operatorname{center}(A)$ such that $\lambda \bar{\lambda}=1$, then we let $\underline{\underline{Q}}^{\lambda}(A)_{\text {even-free }}$ denote the category of nonsingular quadratic 
forms (with minimum form parameter) on finitely generated free modules of even dimension, whose associated sesquilinear form is $\lambda$ Hermitian. We may assume that the modules under consideration have dimension because by definition their equivariant signature vanishes. Let $\mathbb{H}(A)$ denote the hyperbolic plane, cf. [Ba2, §1B]. Define

$$
K Q_{0}^{\lambda}(A)_{\text {even-free }}=K_{0}\left(\underline{\underline{Q}}^{\lambda}(A)_{\text {even-free }}\right) .
$$

If $\mathbb{Z}[H]$ and $\Lambda[H]$ have involutions inverting each element of $H$ then by definition

$$
L_{0}^{h}(\mathbb{Z}[H], 1)=K Q_{0}^{1}(\mathbb{Z}[H])_{\text {even-free }} /[\mathbb{H}(\mathbb{Z}[H])]
$$

and

$$
L_{0}^{h}(\Lambda[H], 1)=K Q_{0}^{1}(\Lambda[H])_{\text {even-free }} /[\mathbb{H}(\Lambda[H])]
$$

Clearly,

$$
\begin{aligned}
\operatorname{coker}\left(L_{0}^{h}(\mathbb{Z}[H]\right. & \left., 1) \rightarrow L_{0}^{h}(\Lambda[H], 1)\right) \\
& =\operatorname{coker}\left(K Q_{0}^{1}(\mathbb{Z}[H])_{\text {even-free }} \rightarrow K Q_{0}^{1}(\Lambda[H])_{\text {even-free }}\right) .
\end{aligned}
$$

Let $A$ be as above and let $X \subset K_{1}(A)$ be an involution invariant subgroup of $K_{1}(A)$ such that $[ \pm \lambda] \in X$. Let $\underline{\underline{Q}}^{\lambda}(A)_{\text {even-free-X }}$ denote the category whose objects are nonsingular quadratic forms on finitely generated free $A$-modules of even rank such that the associated sesquilinear form is $\lambda$-Hermitian and has discriminant in $X$, and whose morphisms are invertible linear maps whose determinant lies in $X$, cf. [B1, $\S 1 \mathrm{~B}]$. Define

$$
K Q_{0}^{\lambda}(A)_{\text {even-free- } X}=K_{0}\left(\underline{\underline{Q}}^{\lambda}(A)_{\text {even-free- } X}\right) .
$$

Clearly,

$$
\underline{\underline{Q}}_{0}^{\lambda}(A)_{\text {even-free }}=\underline{\underline{Q}}_{0}^{\lambda}(A)_{\text {even-free- } K_{1}(A)}
$$

and thus

$$
K Q_{0}^{\lambda}(A)_{\text {even-free }}=K Q_{0}^{\lambda}(A)_{\text {even-free- } K_{1}(A)} \text {. }
$$

Suppose $m$ is a natural number, $S$ the multiplicative set $\{1, m$, $\left.m^{2}, \ldots,\right\}$ and $\Lambda=S^{-1} \mathbb{Z}$. Consider the localization-approximation fibre square of rings with involution

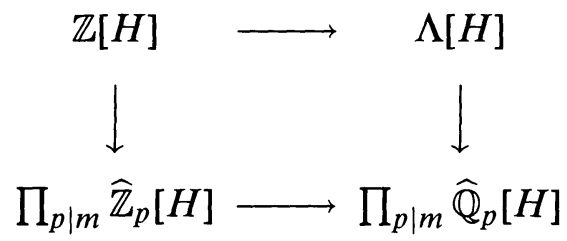

cf. [Ba2, (7.17), (7.18), (7.21), (7.22)]. 
Let $X=K_{1}(\mathbb{Z}[H]), Y=K_{1}(\Lambda[H]), \widehat{X}=\operatorname{image}\left(K_{1}(\mathbb{Z}[H]) \rightarrow\right.$ $\left.K_{1}\left(\prod_{p \mid m} \widehat{\mathbb{Z}}_{p}[H]\right)\right)$ and $\widehat{Y}=\operatorname{image}\left(K_{1}(\Lambda[H]) \rightarrow K_{1}\left(\prod_{p \mid m} \widehat{\mathbb{Q}}_{p}[H]\right)\right)$. By [Ba2, (7.30)c)] (which holds for even-based- $X$ forms as well as based$X$ forms), $[\mathbf{B a 2},(7.26) b)]$, and $[\mathbf{B a 2},(6.26)]$ there is an exact MayerVietoris sequence

$$
\begin{aligned}
& K Q_{0}^{1}(\mathbb{Z}[H])_{\text {even-based- } X} \rightarrow \\
& \qquad Q_{0}^{1}(\Lambda[H])_{\text {even-based- } Y} \oplus K Q_{0}^{1}\left(\prod_{p \mid m} \widehat{\mathbb{Z}}_{p}[H]\right)_{\text {even-based- } \widehat{X}} \\
& \rightarrow K Q_{0}^{1}\left(\prod_{p \mid m} \widehat{\mathbb{Q}}_{p}[H]\right)_{\text {even-based- } \widehat{Y}}
\end{aligned}
$$

from which one deduces an exact sequence

$$
\begin{aligned}
K Q_{0}^{1}(\mathbb{Z}[H])_{\text {even-based- } X} & \rightarrow K Q_{0}^{1}(\Lambda[H])_{\text {even-based- } Y} \\
\rightarrow \operatorname{coker}( & K Q_{0}^{1}\left(\prod_{p \mid m} \widehat{\mathbb{Z}}_{p}[H]\right)_{\text {even-based- } \widehat{X}} \\
& \left.\rightarrow K Q_{0}^{1}\left(\prod_{p \mid m} \widehat{\mathbb{Q}}_{p}[H]\right)_{\text {even-based- } \widehat{Y}}\right) .
\end{aligned}
$$

It suffices to show that the preceding cokernel is finite. Since the quotient of (by definition)

$$
\begin{aligned}
& W Q_{0}^{1}\left(\prod_{p \mid m} \widehat{\mathbb{Q}}_{p}[H]\right)_{\text {even-based- } \widehat{Y}} \\
&=K Q_{0}^{1}\left(\prod_{p \mid m} \widehat{\mathbb{Q}}_{p}[H]\right)_{\text {even-based- } \widehat{Y}} /\left[\mathbb{H}\left(\prod_{p \mid m} \widehat{\mathbb{Q}}_{p}[H]\right)\right]
\end{aligned}
$$

it suffices to show that $W Q_{0}^{1}\left(\prod_{p \mid m} \widehat{\mathbb{Q}}_{p}[H]\right)_{\text {even-based }-\widehat{Y}}$ is finite. Consider the exact sequence $[\mathrm{Ba} 2,(8.4)]$

$$
\begin{aligned}
& H^{1}\left(\mathbb{Z} / 2 \mathbb{Z}, K_{1}\left(\prod_{p \mid m} \widehat{\mathbb{Q}}_{p}[H]\right) / \widehat{Y}\right) \rightarrow W Q_{0}^{1}\left(\prod_{p \mid m} \widehat{\mathbb{Q}}_{p}[H]\right)_{\text {even-based- } \widehat{Y}} \\
& \quad \rightarrow W Q_{0}^{1}\left(\prod_{p \mid m} \widehat{\mathbb{Q}}_{p}[H]\right)_{\text {even-based- } K_{1}\left(\prod_{p \mid m} \widehat{\mathbb{Q}}_{p}[H]\right)} \\
& \quad \rightarrow H^{2}\left(\mathbb{Z} / 2 \mathbb{Z}, K_{1}\left(\prod_{p \mid m} \widehat{\mathbb{Q}}_{p}[H]\right) / \widehat{Y}\right) .
\end{aligned}
$$


By a result of Bass [Bs, V (9.2)], determinant

$$
K_{1}\left(\prod_{p \mid m} \widehat{\mathbb{Q}}_{p}[H]\right) \stackrel{\cong}{\longrightarrow} \text { units }\left(\prod_{p \mid m} \widehat{\mathbb{Q}}_{p}[H]\right) \text {. }
$$

Thus

$$
K_{1}\left(\prod_{p \mid m} \widehat{\mathbb{Q}}_{p}[H]\right) / \widehat{Y}=\operatorname{units}\left(\prod_{p \mid m} \widehat{\mathbb{Q}}_{p}[H]\right) / \operatorname{units}(\Lambda[H]) .
$$

We leave it as an exercise for the reader to show that

$$
H^{i}\left(\mathbb{Z} / 2 \mathbb{Z}, \text { units }\left(\prod_{p \mid m} \widehat{\mathbb{Q}}_{p}[H]\right)\right) \text { and } H^{i}(\mathbb{Z} / 2 \mathbb{Z}, \operatorname{units}(\Lambda[H]))
$$

are finite, for $i \geq 1$. Thus, from the long exact cohomology sequence associated to the exact sequence

$$
\begin{aligned}
1 \rightarrow \operatorname{units}(\Lambda[H]) & \rightarrow \text { units }\left(\prod_{p \mid m} \widehat{\mathbb{Q}}_{p}[H]\right) \\
& \rightarrow \operatorname{units}\left(\prod_{p \mid m} \widehat{\mathbb{Q}}_{p}[H]\right) / \operatorname{units}(\Lambda[H]) \rightarrow 1
\end{aligned}
$$

it follows that

$$
H^{i}\left(\mathbb{Z} / 2 \mathbb{Z}, \text { units }\left(\prod_{p \mid m} \widehat{\mathbb{Q}}_{p}[H]\right) / \operatorname{units}(\Lambda[H])\right)
$$

is finite, for $i=1,2$. Thus, $W Q_{0}^{1}\left(\prod_{p \mid m} \widehat{\mathbb{Q}}_{p}[H]\right)_{\text {even-based- } \widehat{Y}}$ is finite if and only if

$$
W Q_{0}^{1}\left(\prod_{p \mid m} \widehat{\mathbb{Q}}_{p}[H]\right)_{\text {even-based }-K_{1}\left(\prod_{p \mid m} \widehat{\mathbb{Q}}_{p}[H]\right)}=W Q_{0}^{1}\left(\prod_{p \mid m} \widehat{\mathbb{Q}}_{p}[H]\right)_{\text {even-free }}
$$

is finite. The proof is complete by showing that the latter group is finite.

The ring $\prod_{p \mid m} \widehat{\mathbb{Q}}_{p}[H]$ factors as a finite product $\prod_{i} F_{i}$ of rings with involution such that $F_{i}$ is either a product $F_{i}=F \times F$ of fields $F$ with involution exchanging the coordinates, or $F_{i}$ is a $p$-adic cyclotomic field $\widehat{\mathbb{Q}}_{p}(\zeta)$ where $p \mid m$ and $\bar{\zeta}=\zeta^{-1}$. Since

$$
W Q_{0}^{1}\left(\prod_{p \mid m} \widehat{\mathbb{Q}}_{p}[H]\right)_{\text {even-free }} \longmapsto \prod_{i} W Q_{0}^{1}\left(F_{i}\right)_{\text {even-free }}
$$

it suffices to show that each group $W Q_{0}^{1}\left(F_{i}\right)_{\text {even-free }}$ is finite. If $F_{i}=$ $F \times F$ then the group above is trivial, since every form is a product 
of hyperbolic planes. In the remaining cases, the group is either the standard Witt group of the $p$-adic field $\widehat{\mathbb{Q}}_{p}$, which is well known to be finite, or the Witt group of Hermitian forms on the cyclotomic field $\widehat{\mathbb{Q}}_{p}[\zeta] \neq \widehat{\mathbb{Q}}_{p}$, which is also well known to be finite.

\section{REFERENCES}

[AB] M. Atiyah and R. Bott, The Lefschetz fixed point theorem for elliptic complexes II, Annals of Math., 88 (1968), 451-491.

[Ba1] A. Bak, The Computation of Surgery Groups of Finite Groups with Abelian 2-Hyperelementary Subgroups, in: Algebraic K-theory, Evanston 1976, Springer Lecture Notes in Math., 551 (1977), 384-409.

[Ba2] _ K-Theory of Forms, Annals of Math. Studies, Princeton University Press Vol. 98, Princeton, N.J., 1981.

[BS] A. Bak and W. Scharlau, Grothendieck and Witt groups of orders and finite groups, Inventiones Math., 23 (1974), 207-240.

[Bs] H. Bass, Algebraic K-Theory, Benjamin, 1968.

[B] G. Bredon, Representations at fixed points of smooth actions of compact groups, Annals of Math., 89 (1969), 515-532.

[CS1] S. Cappell and J. Shaneson, Fixed points of periodic differentiable maps, Inventiones Math., 68 (1982), 1-19.

[CS2] _ Representations at Fixed Points, in: Group Actions on Manifolds, Contemp. Math., 36 (1985), 151-158.

[C1] E. C. Cho, Smith Equivalent Representations of Generalized Quaternion Groups, Thesis, Rutgers University (1984).

[C2] _ , s-Smith equivalent representations of dihedral groups, Pacific J. Math., 135 (1988), 17-28.

[D] K. H. Dovermann, Even Dimensional Smith Equivalent Representations, in: Algebraic Topology, Aarhus 1982, Springer Lecture Notes in Math., 1051 (1983), 587-602.

[DKS] K. H. Dovermann, I. Knop and D. Y. Suh, Topological invariants of real algebraic actions, to appear in Topology and its Applications.

[DP1] K. H. Dovermann and T. Petrie, G-surgery II, Memoirs Amer. Math. Soc., 260 (1982).

[DP2] _ Smith equivalence of representations for odd order cyclic groups, Topology, 24 (1985), 283-305.

[DP3] _ An induction theorem for equivariant surgery, G surgery III, Amer. J. Math., 105 (1983), 1369-1403.

[DPS] K. H. Dovermann, T. Petrie and R. Schultz, Transformation groups and fixed point data, in: Group Actions on Manifolds, Contemp. Math., 36 (1985), 159-189.

[DR] K. H. Dovermann and M. Rothenberg, Equivariant surgery and classification of finite group actions on manifolds, Memoirs Amer. Math. Soc., 397 (1988).

[DW] K. H. Dovermann and L. D. Washington, Relations between cyclotomic units and Smith equivalence of representations, Topology, 28 (1989), 81-89.

[MP] M. Masuda and T. Petrie, Lectures on transformation groups and Smith equivalence, in: Group Actions on Manifolds, Contemp. Math., 36 (1985), 191-242.

[M] J. Milnor, Whitehead torsion, Bull. Amer. Math. Soc., 72 (1966), 358-426.

[O] R. Oliver, Fixed point sets of group actions on finite acyclic complexes, Comm. Math. Helv., 50 (1975), 155-177. 
[OP] R. Oliver and T. Petrie, $G C W$ surgery and $\widetilde{K}_{0}(\mathbb{Z}[G])$, Math. Zeitschrift, 179 (1982), 11-42.

[P1] T. Petrie, G surgery I-A survey, in: Algebraic and Geometric Topology, Proceedings, Santa Barbara 1977, Springer Lecture Notes in Math., 664 (1978), 197-223.

[P2] _ Smith equivalence of representations, Math. Proc. Camb. Phil. Soc., 94 (1983), 61-99.

[PR1] T. Petrie and J. Randall, Spherical isotropy representations, Publ. I.H.E.S., 62 (1985), 5-40.

[PR2] _ Transformation Groups on Manifolds, Dekker Series in Pure and Applied Mathematics Vol. 82, Marcel Dekker, New York, 1984.

[Sz] C. Sanchez, Actions of groups of odd order on compact orientable manifolds, Proc. Amer. Math. Soc., 54 (1976), 445-448.

[S] J. P. Serre, Linear Representations of Finite Groups, Graduate Texts in Mathematics Vol. 42, Springer Verlag, New York Heidelberg Berlin, 1977.

[Si] A. Siegel, Fixed Point Representations of Group Actions on Spheres, Thesis, Rutgers University (1982).

[Sm] P. A. Smith, New results and old problems in finite transformation groups, Bull. Amer. Math. Soc., 66 (1960), 401-415.

[Su1] D. Y. Suh, s-Smith Equivalence of Representations, Thesis, Rutgers University 1984.

[Su2] _ Isotropy representations of cyclic group actions on homotopy spheres, Bull. Korean Math. Soc., 25 (1988), 175-178.

[Sw] R. Swan, K-Theory of Finite Groups and Orders, Lecture Notes in Mathematics Vol. 149, Springer Verlag, Berlin Heidelberg New York, 1970.

[W] C. T. C. Wall, Surgery on Compact Manifolds, Academic Press, New York, 1970.

Received September 5, 1989. Much of this paper was written during a stay of the first named author at the Max Planck Institut für Mathematik in Bonn, and he would like to thank the Institut for the hospitality. The second author was partially supported by Korea Science and Engineering Foundation.

UNIVERSITY OF HAWAII

HoNOlulu, HI 96822

AND

Korea Institute of TeCHNOLOGY

TAEJON 305-701, KoREA 


\title{
PACIFIC JOURNAL OF MATHEMATICS EDITORS
}

\author{
V. S. VARADARAJAN \\ (Managing Editor) \\ University of California \\ Los Angeles, CA 90024-1555-05 \\ Herbert Clemens \\ University of Utah \\ Salt Lake City, UT 84112 \\ F. Michael Christ \\ University of California \\ Los Angeles, CA 90024-1555 \\ THOMAS ENRIGHT \\ University of California, San Diego \\ La Jolla, CA 92093
}

Nicholas ERcolani

University of Arizona

Tucson, AZ 85721

R. FINN

Stanford University

Stanford, CA 94305

VAUGHAN F. R. JoNeS

University of California

Berkeley, CA 94720

C. C. MOORE

University of California

Berkeley, CA 94720

MARTIN SchaRLEMANN

University of California

Santa Barbara, CA 93106

HAROLd StaRK

University of California, San Diego

La Jolla, CA 92093

SteVen Kerckhoff

Stanford University

Stanford, CA 94305

\section{ASSOCIATE EDITORS}

\begin{tabular}{|c|c|c|c|c|}
\hline R. ARENS & $\begin{array}{l}\text { E. F. BECKENBACH } \\
(1906-1982)\end{array}$ & NeumanN & $\begin{array}{c}\text { F. WoLF } \\
(1904-1989)\end{array}$ & K. YoshidA \\
\hline \multicolumn{5}{|c|}{ SUPPORTING INSTITUTIONS } \\
\hline \multicolumn{2}{|c|}{ JNIVERSITY OF ARIZONA } & \multicolumn{3}{|c|}{ UNIVERSITY OF OREGON } \\
\hline \multicolumn{2}{|c|}{ UNIVERSITY OF BRITISH COLUMBIA } & \multicolumn{3}{|c|}{ UNIVERSITY OF SOUTHERN C } \\
\hline \multicolumn{2}{|c|}{ CALIFORNIA INSTITUTE OF TECHNOLOGY } & \multirow{2}{*}{\multicolumn{3}{|c|}{ STANFORD UNIVERSITY }} \\
\hline \multicolumn{2}{|c|}{ UNIVERSITY OF CALIFORNIA } & & & UNIVERSITY OF HAWAII \\
\hline \multicolumn{2}{|c|}{ MONTANA STATE UNIVERSITY } & \multicolumn{3}{|c|}{ UNIVERSITY OF TOKYO } \\
\hline \multicolumn{2}{|c|}{ UNIVERSITY OF NEVADA, RENO } & \multicolumn{3}{|c|}{ UNIVERSITY OF UTAH } \\
\hline \multicolumn{2}{|c|}{ NEW MEXICO STATE UNIVERSITY } & \multicolumn{3}{|c|}{ DN STATE UNIVERSITY } \\
\hline \multicolumn{2}{|c|}{ OREGON STATE UNIVERSITY } & UNIVER & OF WASHIN & \\
\hline
\end{tabular}




\section{Pacific Journal of Mathematics}

Vol. 152, No. $1 \quad$ January, 1992

B. V. Rajarama Bhat, On a characterization of velocity maps in the space of observables .......................................

John David Brillhart, Note on the discriminant of certain cyclotomic period

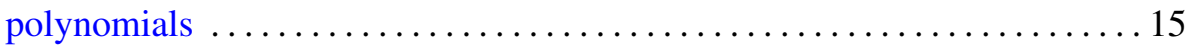

Steven R. Costenoble and Stefan Waner, The equivariant Thom

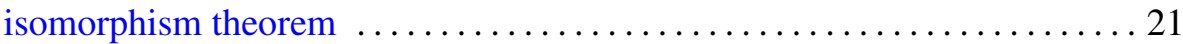

Karl Heinz Dovermann and Dong Youp Suh, Smith equivalence for finite

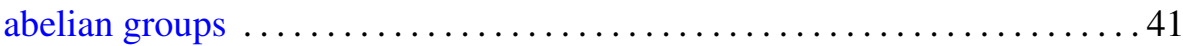

Klaus-Jochen Engel, On singular perturbations of second order Cauchy

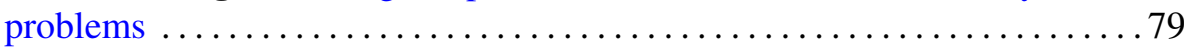

Angel Ferrandez and Pascual Lucas, On surfaces in the 3-dimensional

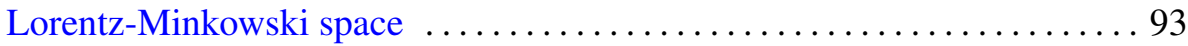

Brian Hartley, A general Brauer-Fowler theorem and centralizers in locally

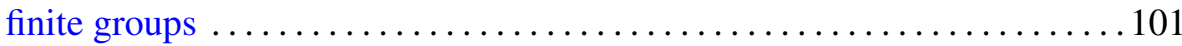

Joel Hass, Intersections of least area surfaces .................... 119

Edward Kissin, Indices of unbounded derivations of $C^{*}$-algebras $\ldots \ldots \ldots 125$

Erhard Luft and Denis Karmen Sjerve, On regular coverings of

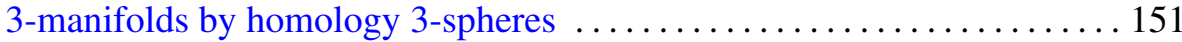

Daniel Pecker, Sur la projection de variétés algébriques réelles .......... 165

Claus Scheiderer, Some remarks on orderings under finite field

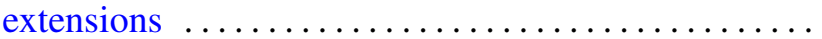

Fernando Serrano, Elliptic surfaces with an ample divisor of genus two 